Travelling wave solutions for the Richards equation incorporating non-equilibrium effects in the capillarity pressure Non Peer-reviewed author version

C.J van Duijn; MITRA, Koondanibha \& POP, Sorin (2018) Travelling wave solutions for the Richards equation incorporating non-equilibrium effects in the capillarity pressure. In: NONLINEAR ANALYSIS-REAL WORLD APPLICATIONS, 41, p. 232-268.

DOI: 10.1016/j.nonrwa.2017.10.015

Handle: http://hdl.handle.net/1942/25330 


\section{UHASSELT}

UHasselt Computational Mathematics Preprint Series

\section{Travelling wave solutions for the Richards equation incorporating non-equilibrium effects in the capillarity pressure}

Hans van Duijn, Koondanibha Mitra and Iuliu Sorin Pop

UHasselt Computational Mathematics Preprint Nr. UP-17-06

July 5,2017 


\title{
Travelling wave solutions for the Richards equation incorporating non-equilibrium effects in the capillarity pressure
}

\author{
C.J. van Duijn ${ }^{1,2}$, K. Mitra*3,4, and I.S. Pop ${ }^{4,5}$ \\ ${ }^{1}$ Eindhoven University of Technology, Department of Mechanical Engineering \\ ${ }^{2}$ University of Utrecht, Department of Earth Sciences \\ ${ }^{3}$ Eindhoven University of Technology, Department of Mathematics and Computer Science \\ ${ }^{4}$ Hasselt University, Faculty of Science \\ ${ }^{5}$ University of Bergen, Department of Mathematics
}

\begin{abstract}
The Richards equation is a mathematical model for the unsaturated flow through porous media. This paper considers an extension of the Richards equation, where non-equilibrium effects like hysteresis and dynamic capillarity are incorporated in the relationship that relates the water pressure and the saturation. The focus is on travelling wave solutions, for which the existence is investigated first for the model including hysteresis and subsequently for model including dynamic capillarity effect. In particular, such solutions may have non monotonic profiles, which are ruled out when considering standard, equilibrium type models, but have been observed experimentally. The paper ends with numerical experiments confirming the theoretical results. In this sense the original system of partial differential equations is solved by means of an implicit scheme. This relies on an equivalent, mixed formulation of the system. For solving the resulting nonlinear, time-discrete problems, a linear iterative scheme is proposed.
\end{abstract}

\section{Introduction}

Unsaturated flow through porous media is encountered in many applications of societal and engineering relevance. Examples in this sense are the groundwater flows, or the moisture dynamics in building materials. A commonly used mathematical model for such kind of processes is the Richards equation, which is obtained after inserting the Darcy law into the water mass balance equation. The two main unknowns in this equation are the water saturation $S$ (the percentage of the pore space in a representative elementary volume that is occupied by water) and the water pressure $p$. In standard porous media flow models, these two unknowns are related through the strictly decreasing capillary pressure function $P_{c}(\cdot)$, namely $p=-P_{c}(S)$, which is determined experimentally. Different types of functions and parameterizations are discussed e.g. in [31], the common assumption being that these dependencies are obtained under special, equilibrium conditions. More precisely, the experiments are carried out either for imbibition or for draining and not when these processes occur alternatively, and during an entire imbibition or drainage cycle each measurement has been done only after water stops redistributing inside the pores of the elementary volumes. Such models will therefore be called in what follows "equilibrium type models".

*Corresponding author: email: k.mitra@tue.nl 
In realistic applications, neither of these conditions are met. First, experiments reported e.g. in $[15,29]$ have revealed the hysteretic nature of the pressure - saturation relationship. More precisely, it was observed that the functions $P_{c}$ determined during infiltration and drainage are different. This motivated an extremely rich literature on mathematical models describing hysteresis. The play-type hysteresis model assumes a switch between imbibition and drainage capillary pressure-saturation curves whenever the saturation changes from increasing in time to decreasing in time or vice-versa. A mathematical formulation of this is given in [3], and the switch happens along vertical scanning curves. This poses nontrivial issues when analysing the resulting models and their numerical discretisations, which can be resolved by approximating the vertical scanning curves by monotone and non-vertical ones. In this sense, commonly used is the Lenhard-Parker model [33], where the scanning curves are rescaled versions of some predefined curves. A simplified version of it is proposed in [10], where the scanning curves are oblique lines. Other hysteresis models build on concepts like percolating/nonpercolating phases $[20,24]$, or interfacial area based models $[30,35]$. An overview of hysteresis models can be found in [50], whereas details on the numerical approximation of hysteresis in porous media models are given in [32]. In the present paper we consider the play-type hysteresis model for the pressure - saturation dependency but it is interesting to note that hysteresis can also be present in the relative permeability curve [34]. However, in the latter case this effect is less important in comparison to the former [15].

Second, when letting the water infiltrate in a homogeneous medium, experiments have revealed profiles that are conflicting with the profiles of the solutions to the equilibrium type models. For example, if the injection rates at the inflow are high enough, the obtained saturation profiles are non-monotone as the values at some locations inside the column are higher than at the inflow boundary (the so-called overshoot phenomenon, see [18]). In particular, the experiments in [7] show that although the saturation at some certain location is decreasing in time, the water pressure is non-monotone and exhibits a peak at moments when the saturation changes rapidly. This pleads for the inclusion of dynamic effects in the pressure-saturation relationship, as suggested in [22].

In mathematical terms, models like those mentioned above are evolution equations of pseudoparabolic type, or involve differential inclusions. Such models will be called below "nonequilibrium type models". In this paper, we investigate how the solution profiles for unsaturated flow through a long, homogeneous porous column are affected by such non-equilibrium effects. The analysis is based on travelling waves (TW), allowing to reduce the model first to a nonlinear ordinary differential equation, and then to a dynamical system. This provides insight in the structure and behaviour of the solutions, and in particular how the non-equilibrium regime affects the profiles. The present analysis follows the ideas in [46], which studies the existence of TW solutions for reactive flow and transport models in porous media. In [17] TW solutions are analysed for nonlinear models that are similar to the Richards equation, but where higher order effects are included inspired by the ones describing dynamic capillarity. The nonlinear functions taken in [17] are of power-like type, in particular the flux function is convex. The existence of TW solutions is analysed, and in particular it is shown that oscillations behind the infiltration front may occur, depending on the magnitude of the dynamic effect. A similar analysis, but for two-phase flow models implying convex-concave flux functions is carried out in [44, 47, 48]. Also related are the diffusive-dispersive equations appearing as models for the phase transition dynamics, but in which the higher order terms are in terms of the spatial derivatives only $[2,16]$. Though having a different motivation, the associated TW equation is similar to the one for the dynamic capillarity models, in particular since both involve a non-convex nonlinearity in the lower order terms. In this context, in [47] it is proved that the saturation profile may have overshoot in form of a plateau separated by two fronts (infiltration-drainage), similar to the ones 
obtained in [18]. The dependence of the saturation value at such plateaus on the magnitude of the dynamic effect is proved rigorously in [47], and non-standard entropy conditions are defined for the shock solutions of the limiting hyperbolic case when the capillary effects are neglected. This analysis is extended to the case of degenerate models in $[44,48]$. Due to the degeneracy in the model, the saturation remains between the physically relevant values, but the TW solutions may have discontinuous derivatives. The possibility of encountering non-monotonic TW profiles for various extensions of the Richards equation, including dynamic capillarity models, is evidenced numerically in [19, 21]. Finally, we mention [51] for a numerical study of the saturation and capillary pressure profiles for several of the hysteresis concepts discussed above, combined with dynamic capillarity.

The present analysis consists of three parts. First the existence of TW solutions is analysed for the models involving hysteresis. The TW profiles are obtained by regularising the multi-valued function involved in the hysteretic term. In particular, we analyse the orbits associated with the TW solution in the saturation-pressure plane. We prove that in the initial and the final stages these orbits follow scanning curves that become vertical when the regularisation parameter vanishes, and in between they follow the corresponding primary curves (imbibition/drainage).

Next, the case where dynamic effects are present in the pressure - saturation relationship is discussed. The existence of TW solutions is obtained and criteria ensuring their nonmonotonicity are provided. These include also situations where full-saturation is achieved. In the last part we discuss a numerical scheme for approximating the solution of the non-linear, pseudo-parabolic partial differential equations modelling the processes described above. The scheme is implicit, so at each time step one has to solve a nonlinear problem. In this context we propose an iterative method which is unconditionally convergent. Finally, numerical results validating the theoretical findings are provided. As will be seen below, the numerical solutions to the original model are reproducing nicely analytically predicted structures and properties of the TW solutions.

\section{Mathematical formulation}

\subsection{Basic equations}

We consider the unsaturated water flow in a one-dimensional, homogeneous porous medium. Let $t$ and $x$ denote the time and space variable respectively. Assuming that the medium is vertical so that gravity effects are playing a role, a well accepted model for the flow is the Richards equation [31],

$$
\phi \frac{\partial S}{\partial t}=\frac{\partial}{\partial x}\left[\kappa \frac{k(S)}{\mu} \cdot\left(\frac{\partial p}{\partial x}-\rho g\right)\right] .
$$

The unknowns in the model are the water saturation $S$ and the water pressure $p$. The relative permeability $k(\cdot)$ is a given, positive and increasing function that characterizes the medium and can be determined experimentally. The other quantities are parameters in the model and are assumed positive and known: $\mu$ and $\rho$ are the the water viscosity and density, $g$ is the gravitational acceleration, $\phi$ is the porosity of the medium, and $\kappa$ its absolute permeability.

The model is completed by a an equation describing the dependency between $p$ and $S$. For standard models, this dependency is algebraic,

$$
-p=P_{c}(S)
$$


where $P_{c}(\cdot)$ is a decreasing function. Its specific form is determined experimentally. As mentioned, the results available in the literature assume a local equilibrium and disregard the history of the system.

Here we consider the non-equilibrium model proposed in [3], which combines dynamic effects in the $p$ - $S$ relationship with a simple, play type hysteresis model. For a mathematical justification of the play-type hysteresis, based on the pore scale analysis, we refer to [43]. Let $p_{i m b}(\cdot)$ and $p_{d r n}(\cdot)$ be the primary imbibition and drainage capillary pressure curves [29] respectively. In the absence of the dynamic effects one has

$$
P_{c}(S)=\left\{\begin{array}{l}
p_{i m b}(S) \text { for } \partial_{t} S>0 \text { (infiltration) } \\
p_{d r n}(S) \text { for } \partial_{t} S<0 \text { (drainage) }
\end{array}\right.
$$

Combining this with the vertical scanning curves the closure relationship can be written in the compact mathematical form

$$
-p=P_{c}\left(S, \frac{\partial S}{\partial t}\right) \in P^{+}(S)-P^{-}(S) \cdot \operatorname{sign}\left(\frac{\partial S}{\partial t}\right)
$$

where $\operatorname{sign}(\cdot)$ is the multi-valued function (the signum graph)

$$
\operatorname{sign}(u)= \begin{cases}1 & \text { for } u>0 \\ {[-1,1]} & \text { for } u=0 \\ -1 & \text { for } u<0\end{cases}
$$

The functions $P^{+}, P^{-}$are defined as (also see Figure 1 for an example in the dimensionless framework)

$$
P^{+}=\frac{1}{2}\left(p_{d r n}+p_{i m b}\right), \quad \text { and } \quad P^{-}=\frac{1}{2}\left(p_{d r n}-p_{i m b}\right) .
$$

To include the dynamic effects we refer to [22]. With $\tau$ being a damping parameter and $f(\cdot)$ a damping function (both non-negative), the model combining hysteretic and dynamic effects in the pressure-saturation relationship reads

$$
-p \in P^{+}(S)-P^{-}(S) \cdot \operatorname{sign}\left(\frac{\partial S}{\partial t}\right)-\tau f(S) \frac{\partial S}{\partial t} .
$$

In [22], a thermodynamic justification of such models has been given. Also, homogenisation techniques are employed in [8] for justifying the dynamic terms. For experimental studies concerning the value of $\tau$ and the shape of the function $f$ we refer to [7].

\subsection{Scaling and assumptions}

In what follows we assume that water infiltrates in a porous column under both capillary and gravity effects. The column is assumed isotropic and homogeneous, implying that $\phi$ and $\kappa$ are constants. We also assume that the column is insulated laterally, so the flow will be essentially one-dimensional, in the direction of the gravity. Since we consider here TW solutions, the column is assumed infinite.

With $\sigma$ being the air-water surface tension coefficient, we consider the reference quantities

$$
p^{*}=\sigma \sqrt{\frac{\phi}{\kappa}}, \quad L^{*}=\frac{p^{*}}{\rho g}, \quad T^{*}=\frac{\mu \phi L}{\rho g \kappa}, \quad \tau^{*}=\frac{\mu L^{2} \phi}{\kappa},
$$


and apply the rescaling

$$
\tilde{x}=\frac{x}{L^{*}}, \quad \tilde{p}=\frac{p}{p^{*}}, \quad \tilde{t}=\frac{t}{T^{*}} \quad \tilde{\tau}=\frac{\tau}{\tau^{*}} .
$$

Observe that reference value for pressure is inspired by the J-Leverett relationship and the reference value for the damping parameter $\tau^{*}$ is consistent with [26]. Also, since the analysis below will involve infinite domains, we have first specified a reference pressure and based on it a reference length has been defined. Putting the scaled variables in (2.1) and (2.6) and disregarding the ${ }^{\sim}$ to simplify the notation one obtains the dimensionless system

$$
\begin{aligned}
& \frac{\partial S}{\partial t}=\frac{\partial}{\partial x}\left[k(S)\left(\frac{\partial p}{\partial x}-1\right)\right], \\
& -p \in P^{+}(S)-P^{-}(S) \cdot \operatorname{sign}\left(\frac{\partial S}{\partial t}\right)-\tau f(S) \frac{\partial S}{\partial t} .
\end{aligned}
$$

Next we state the assumptions on the nonlinear functions involved in the model. The assumptions are justified from physical point of view.

(A. 1) The relative permeability $k \in C^{1}([0,1])$ is a continuously differentiable, increasing, bounded and convex function. There exists $M_{k}>0$ s.t. for all $S \in[0,1]$ one has $0 \leq k(S) \leq 1$, and $0 \leq k^{\prime}(S) \leq M_{k}$

(A. 2) The damping parameter is positive, $\tau \geq 0$. The damping function is continuous and satisfies $f(S)>0$ for all $S \in(0,1)$.

(A. 3) The scaled primary drainage and imbibition pressure functions $p_{i m p}, p_{d r n}:(0,1] \rightarrow[0, \infty)$ are continuously differentiable, strictly decreasing and satisfy

$$
p_{i m b}(1)=p_{d r n}(1)=0, \quad \text { and } \quad p_{i m b}(S)<p_{d r n}(S) \text { for all } S \in(0,1) \text {; }
$$

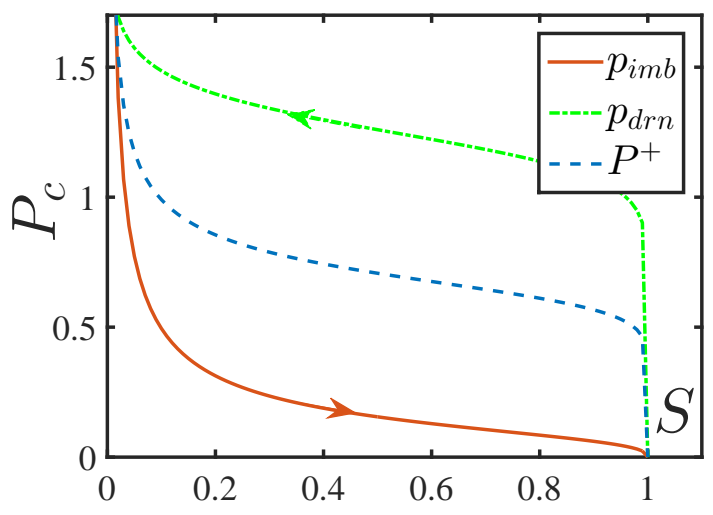

Figure 1: Dimensionless primary imbibition $\left(p_{i m b}\right)$ and drainage $\left(p_{d r n}\right)$ capillary pressure curves and their average $\left(P^{+}\right)$as a function of saturation $S$. The curves are based on the van Genuchten model [49] and the parameters are taken from experiments [52, p. 91].
An immediate consequence is that the functions $P^{ \pm}(\cdot)$ defined in $(2.5)$ are in $C^{1}((0,1])$, satisfying $P^{ \pm}(1)=0$ and $P^{ \pm}(S)>0$ for all $S \in(0,1)$. Also, for any $\delta>0$, a constant $M_{P}(\delta)>0$ exists s.t. $-M_{P}(\delta)<\left(P^{+}\right)^{\prime}(S)<0$ for all $S \in[\delta, 1]$. Figure 1 displays an example of primary drainage and imbibition curves and their average $P^{+}$.

To analyse the effect of hysteresis, which is modelled by means of a multivalued function, we consider a regularisation approach. With $\varepsilon>0$ being a small regularisation parameter, one can approximate the sign function by another function $H_{\varepsilon}: \mathbb{R} \rightarrow \mathbb{R}$ satisfying the following

(A. 4) For each $\varepsilon>0, H_{\varepsilon}$ is smooth, odd and strictly increasing. It satisfies

$$
H_{\varepsilon}(-s)=-H_{\varepsilon}(s) \text { and } 0<H_{\varepsilon}^{\prime}(s) \leq H_{\varepsilon}^{\prime}(0)=\frac{1}{\varepsilon} \text { for all } s \in \mathbb{R} ;
$$


(A. 5) $H_{\varepsilon}$ is monotone w.r.t. $\varepsilon$ : if $\varepsilon_{1}>\varepsilon_{2}>0$ then $\left|H_{\varepsilon_{1}}(s)\right|<\left|H_{\varepsilon_{2}}(s)\right|<1$ for all $s \neq 0$. Also, when $\varepsilon \searrow 0$, the functions $H_{\varepsilon}$ are approaching the signum graph:

$$
\lim _{\varepsilon \rightarrow 0} H_{\varepsilon}(s)=-1 \text {, if } s<0 \text {, and } \lim _{\varepsilon \rightarrow 0} H_{\varepsilon}(s)=1 \text {, if } s>0 .
$$

Finally, $H_{\varepsilon}$ depends on $\varepsilon$ in a continuously differentiable manner.

Observe that $H_{\varepsilon}(0)=0$ for all $\varepsilon>0$, that $H_{\varepsilon}(s)$ decreases with $\varepsilon$ for negative arguments $s$, and that this monotonicity is reversed for positive arguments.

When sign is replaced by $H_{\varepsilon}$ in $(2.10)$, the regularised model for the pressure-saturation relationship becomes

$$
-p=P^{+}(S)-P^{-}(S) H_{\varepsilon}\left(\frac{\partial S}{\partial t}\right)-\tau f(S) \frac{\partial S}{\partial t} .
$$

Such regularisation has been used in $[39,42]$ for proving the existence of weak solutions to such models, and for developing appropriate numerical schemes.

It is an intriguing question whether this approach is motivated physically, and in particular how the parameter $\varepsilon$ can be interpreted from physical point of view. In this sense one observes that in the play type hysteresis models, a switch from drainage to imbibition leads to a vertical jump between the corresponding primary curves in the pressure-saturation plane. In physical terms, the switch between the primary curves is made along vertical scanning curves. When considering regularised models, these scanning curves have a steep but finite gradient. This is actually observed in scanning curves obtained from experiments in [29]. As will be seen below, the same profiles are predicted by the TW analysis, and the scanning curves become steeper when $\varepsilon$ is decreased.

Another motivation for considering regularised models can be found in [50], where the play type hysteresis is viewed as a 'friction-controlled backslash' process. This means that dissipative forces, which are mostly continuous in porous media, are responsible for it. At the pore scale, hysteresis occurs because of the difference in the advancing and receding contact angles of the wetting phase, which is a continuous phenomenon and hence jump phenomena should not be expected.

Based on the above, $\varepsilon$ can be seen as a physical parameter, or at least can be used to fit more realistic $P_{c}-S$ scanning curves. Having this in mind, in the subsequent discussions we will analyse first the case $\varepsilon>0$ and then the limiting case of $\varepsilon \rightarrow 0$. Before doing so we mention that (2.9), combined with the constitutive relationship (2.10) or its regularised counterpart (2.12), becomes a nonlinear, pseudo-parabolic equation. In general, one cannot expect that solutions exist in a classical sense. We refer to $[5,6,12,13,14,25,28,27,41,42,43]$ for results concerning the existence and uniqueness of weak solutions for hysteresis models, dynamic capillarity models, or for models including both effects. In particular we refer to [41, 42, 13] where, as suggested in $[3,4],(2.12)$ is used to express $\partial_{t} S$ as a function of $S$ and $p$. We rely on the same idea for the TW analysis below.

\subsection{Travelling wave formulation}

To simplify the analysis and to understand the profile of the solutions to the regularised mathematical model (2.9), (2.12) we look for TW solutions. We assume that the solutions have profiles that do not change in time, but travel with a velocity $c$ that will be determined later. Specifically, we extend the domain (the porous medium) to the entire real axis $\mathbb{R}$ and assume that the saturation and the pressure depend on the TW variable $\zeta=c t-x$. Note that this choice is the opposite of $x-c t$, which is commonly used in the TW analysis, but it is convenient 
for the analysis below. For the ease of presentation, we define the negative pressure $u=-p$, and assume that $S$ and $p$ only depend on $\zeta$,

$$
S(x, t)=S(\zeta) \quad \text { and } \quad p(x, t)=-u(\zeta), \quad \text { with } \zeta=c t-x .
$$

The wave velocity $c \in \mathbb{R}$ will be determined below. With the travelling wave transformation (2.9) and (2.12) become

$$
\begin{aligned}
c S^{\prime} & =\left(k(S)\left(u^{\prime}+1\right)\right)^{\prime}, \\
u & =P^{+}(S)-P^{-}(S) H_{\varepsilon}\left(c S^{\prime}\right)-c \tau f(S) S^{\prime},
\end{aligned}
$$

defined for all $\zeta \in \mathbb{R}$. In the above equations ' denotes the differentiation w.r.t. $\zeta$.

Furthermore, we assume that the saturation and the pressure admit horizontal asymptotes at $\pm \infty$,

$$
\begin{array}{ll}
\lim _{\zeta \rightarrow-\infty} S(\zeta)=S_{B}, & \lim _{\zeta \rightarrow \infty} S(\zeta)=S_{T}, \\
\lim _{\zeta \rightarrow-\infty} u(\zeta)=p_{B}, & \lim _{\zeta \rightarrow \infty} u(\zeta)=p_{T} .
\end{array}
$$

for given saturations $S_{T}, S_{B}$ satisfying $S_{T}>S_{B}>0$ and 'pressures' $p_{T}$ and $p_{B}$. Observe that since $u=-p, p_{T}$ and $p_{B}$ are the additive inverses of the actual pressure.

After integrating (2.14), we use the behaviour of $S$ and $u$ at $\pm \infty$ to deduce that

$$
\lim _{\zeta \rightarrow \pm \infty} u^{\prime}(\zeta)=\lim _{\zeta \rightarrow \pm \infty} S^{\prime}(\zeta)=0
$$

Moreover, from (2.15) one obtains that

$$
\lim _{\zeta \rightarrow-\infty} u(\zeta)=P^{+}\left(S_{B}\right) \text { and } \lim _{\zeta \rightarrow \infty} u(\zeta)=P^{+}\left(S_{T}\right),
$$

which provides a necessary condition for the existence of TW solutions. We have

Proposition 2.1 A necessary condition for the existence of $T W$ solutions is that the components in the left and right states are compatible, namely $p_{\alpha}=P^{+}\left(S_{\alpha}\right)(\alpha \in\{T, B\})$.

In what follows, this compatibility condition is assumed unless stated otherwise.

Integrating (2.14) and using the boundary conditions to determine the wave speed and the constant of integration gives

$$
u^{\prime}=\mathcal{G}\left(S ; S_{B}, S_{T}\right) \text { and } c=\frac{k\left(S_{T}\right)-k\left(S_{B}\right)}{S_{T}-S_{B}} .
$$

The function $\mathcal{G}:(0,1] \rightarrow \mathbb{R}$ depends on the parameters $S_{B}$ and $S_{T}$ satisfying $0<S_{B}<S_{T}<1$, and is defined as

$$
\mathcal{G}\left(S ; S_{B}, S_{T}\right):=1-\frac{c\left(S-S_{B}\right)+k\left(S_{B}\right)}{k(S)}=1-\frac{c\left(S-S_{T}\right)+k\left(S_{T}\right)}{k(S)} .
$$

The last equality follows from the definition of the wave speed $c$.

In the subsequent analysis we investigate the TW solutions for hysteresis $(\tau=0)$ and dynamic capillarity cases $\left(p_{i m b}(S)=p_{d r n}(S)\right.$ for $0<S \leq 1$ implying $\left.P^{-}(S)=0\right)$ separately.

Recalling (2.16)-(2.17) and Proposition 2.1, the points $E_{\alpha}=\left(S_{\alpha}, p_{\alpha}\right)$ with $p_{\alpha}=P^{+}\left(S_{\alpha}\right)$ $(\alpha \in\{T, B\})$ and $S_{T}>S_{B}$ are equilibria for the dynamical system (2.14)-(2.15). Therefore, to find and analyse TW solutions of the non-equilibrium model (2.9), (2.12) we seek orbits 
connecting the points $E_{B}$ and $E_{T}$ in the $S$ - $u$ plane. Clearly, corresponding waves are unique up to a translation in the $\zeta$ coordinate. To fix the orbit we also assume

$$
S(0)=\frac{1}{2}\left(S_{B}+S_{T}\right) \text { and } S(\zeta)<\frac{1}{2}\left(S_{B}+S_{T}\right) \text { for all } \zeta<0 .
$$

We will see later, the inequality in $(2.20)$ is needed as $S$ will become non-monotonic if the equilibrium point $E_{T}$ becomes a stable spiral sink. From now on while discussing travelling waves or orbits we implicitly assume that (2.20) is satisfied.

\section{Capillary hysteresis}

We start the analysis by considering first only hysteretic effects in the absence of dynamic ones $(\tau=0)$ in the capillary pressure. Since $H_{\varepsilon}$ is invertible, one can define the function

$$
\Phi_{\varepsilon}:(-1,1) \rightarrow \mathbb{R}, \quad \Phi_{\varepsilon}(r)=\frac{1}{c} H_{c \varepsilon}^{-1}(r),
$$

where $c$ is the wave speed in (2.18). The particular choice of $H_{c \varepsilon}^{-1}$ instead of $H_{\varepsilon}^{-1}$ is made to simplify the writing below. Recalling the Assumptions (A.4) and (A.5) one gets

Proposition $3.1 \Phi_{\varepsilon}$ is a smooth, odd and increasing function satisfying $\Phi_{\varepsilon}^{\prime}(0)=\varepsilon$ for all $\varepsilon>0$. Also, given two regularisation parameters $\varepsilon_{1,2}$ s.t. $\varepsilon_{2}>\varepsilon_{1}>0$ one has $\left|\Phi_{\varepsilon_{1}}(r)\right|<\left|\Phi_{\varepsilon_{2}}(r)\right|$ for all $r \in(-1,1)$. Finally, $\lim _{\varepsilon \rightarrow 0} \Phi_{\varepsilon}(r)=0$ for all $r \in(-1,1)$.

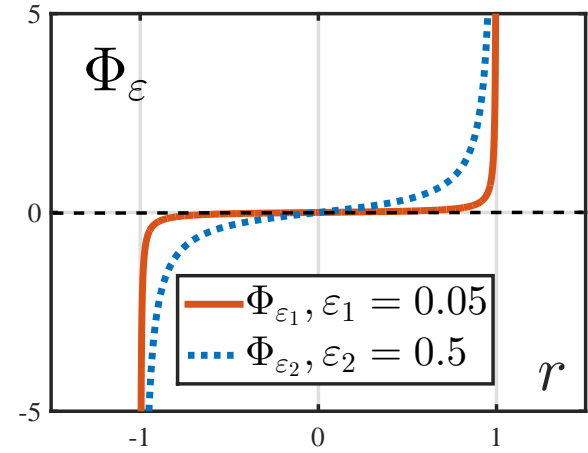

Figure 2: The graph of $\Phi_{\varepsilon}$. The plots are for $\Phi_{\varepsilon}(r)=$ $\frac{\varepsilon r}{\sqrt{1-r^{2}}}$ and two $\varepsilon$ values are $\varepsilon_{1}=0.05$ and $\varepsilon_{2}=0.5$.
The proof is straightforward. Figure 2 shows the graph of $\Phi$ for different values of $\varepsilon$.

Putting $\tau=0$ in (2.15) and rearranging the resultant equation we have the following system

$$
\begin{aligned}
& S^{\prime}=\Phi_{\varepsilon}\left(\frac{P^{+}(S)-u}{P^{-}(S)}\right) \\
& u^{\prime}=\mathcal{G}\left(S ; S_{B}, S_{T}\right) .
\end{aligned}
$$

For the TW analysis we consider the cases $S_{T}=1$ and $S_{T}<1$ separately.

\subsection{The case $S_{T}=1$}

Below we will use the following inequality, which follows directly from Assumption (A.5) and $(2.15)$

$$
-1<\frac{P^{+}(S)-u}{P^{-}(S)}<1 \quad \text { for all } \quad 0<S<1 .
$$

The main result of this section is

Theorem 3.1 Let $0<S_{B}<S_{T}=1$ and $E_{B}=\left(S_{B}, P^{+}\left(S_{B}\right)\right), E_{T}=(1,0)$.

(a) Let $\varepsilon>0$ be fixed. The system (3.2)-(3.3) has a unique orbit $\left(S_{\varepsilon}, u_{\varepsilon}\right)$ connecting the points $E_{B}$ and $E_{T}$. This orbit is increasing in $S$ and decreasing in $u$. Consequently, for any $S \in\left[S_{B}, 1\right]$ there exists a unique $\zeta_{\varepsilon} \in \mathbb{R}$ s.t. $S_{\varepsilon}\left(\zeta_{\varepsilon}\right)=S$, and similar result holds for $u \in\left[0, P^{+}\left(S_{B}\right)\right]$. 
(b) The orbits $\left(S_{\varepsilon}, u_{\varepsilon}\right)$ are well ordered with respect to $\varepsilon$ and do not intersect except at the equilibrium points $E_{B}$ and $E_{T}$. Specifically, if $\varepsilon_{2}>\varepsilon_{1}>0$ and $S_{\varepsilon_{1}}\left(\zeta_{1}\right)=S_{\varepsilon_{2}}\left(\zeta_{2}\right)=S$ for some $S \in\left(S_{B}, 1\right)$ and $\zeta_{1,2} \in \mathbb{R}$, then $u_{\varepsilon_{2}}\left(\zeta_{1}\right)>u_{\varepsilon_{1}}\left(\zeta_{2}\right)$.

(c) Let $S \in\left(S_{B}, 1\right]$ be fixed. For arbitrary $\varepsilon>0$, let $\zeta_{\varepsilon, S} \in \mathbb{R}$ be s.t. $S_{\varepsilon}\left(\zeta_{\varepsilon, S}\right)=S$ and $w_{\varepsilon, S}=u_{\varepsilon}\left(\zeta_{\varepsilon, S}\right)$. Then $\lim _{\varepsilon \rightarrow 0} w_{\varepsilon, S}=p_{i m b}(S)$.

Observe that for any $\varepsilon>0$ Theorem 3.1 provides the existence of TW solutions to the regularised system $(2.9),(2.12)$ that connect the states $\left(S_{B}, P^{+}\left(S_{B}\right)\right)$ and $\left(S_{T}, P^{+}\left(S_{T}\right)\right)=(1,0)$. Here uniqueness is stated under the assumption in (2.20). Thus once the orbit is known, one can let $\varepsilon \searrow 0$ to obtain the existence of TW solutions for the play-type hysteresis model.

Further, due to the monotonic behaviour of the orbits, the TW solutions are monotone in both components and in particular no overshoot is possible in either pressure or saturation. Finally, once the monotonicity is proved, for any fixed $\varepsilon>0$, the functions $S_{\varepsilon}: \mathbb{R} \rightarrow\left(S_{B}, 1\right)$ and $u_{\varepsilon}: \mathbb{R} \rightarrow\left(0, P^{+}\left(S_{B}\right)\right)$ are one to one. This is being used in the last point of Theorem 3.1 when, for arbitrary $S \in\left(S_{B}, 1\right)$, a TW argument $\zeta_{\varepsilon, S}$ and the pressure $w_{\varepsilon, S}$ were defined. Moreover, for any $\zeta<\zeta_{\varepsilon, S}$ one has $S_{\varepsilon}(\zeta)<S$ and $u_{\varepsilon}(\zeta)>w_{\varepsilon, S}$. Similarly for $\zeta>\zeta_{\varepsilon, S}$ one has $S_{\varepsilon}(\zeta)>S$ and $u_{\varepsilon}(\zeta)<w_{\varepsilon, S}$. Recalling $S_{T}=1$ this justifies

Definition 3.1 Let $\varepsilon>0$ and $\left(S_{\varepsilon}, u_{\varepsilon}\right)$ be the orbit of (3.2)-(3.3) connecting the equilibria $E_{B}$ and $E_{T}$. Let $S \in\left(S_{B}, 1\right)$ and $\zeta_{\varepsilon, S} \in \mathbb{R}$ be such that $S_{\varepsilon}\left(\zeta_{\varepsilon, S}\right)=S$. The functions $\eta_{\varepsilon}, w_{\varepsilon}$ : $\left(S_{B}, 1\right) \rightarrow \mathbb{R}$ are defined as

$$
\eta_{\varepsilon}(S)=\zeta_{\varepsilon, S}, \quad w_{\varepsilon}(S)=u_{\varepsilon}\left(\zeta_{\varepsilon, S}\right)
$$

Note that the definition is given for $S \in\left(S_{B}, 1\right)$, but it can be extended to $S=S_{B}$ and $S=1$, with $w_{\varepsilon}$ taking the values $P^{+}\left(S_{B}\right)$, and 0 respectively, and $\eta_{\varepsilon}$ becoming $\mp \infty$. Further, since the definition makes sense whenever $S$ is monotonic, if this does not hold globally, the functions $\eta_{\varepsilon}$ and $w_{\varepsilon}$ can still be defined but restricted to intervals where the saturation is monotone. Finally, from (3.2) and (3.3) one obtains

$$
w_{\varepsilon}^{\prime}(S)=\frac{d w_{\varepsilon}}{d S}(S)=\frac{\mathcal{G}\left(S ; S_{B}, 1\right)}{\Phi_{\varepsilon}\left(\frac{P^{+}(S)-w_{\varepsilon}}{P^{-}(S)}\right)} .
$$

This will be used in the analysis below.

To prove Theorem 3.1 we need some intermediate results. We start with

Proposition 3.2 The region $H^{-}=\left\{(S, u): S_{B} \leq S \leq 1\right.$ and $\left.p_{i m b}(S) \leq u \leq P^{+}(S)\right\}$ is positive invariant for the dynamical system (3.2)-(3.3).

Proof Since $k(\cdot)$ is a convex function it follows that $\mathcal{G}\left(S ; S_{B}, 1\right) \leq 0$ for any $S \in\left[S_{B}, 1\right]$. Also $S_{\varepsilon}^{\prime} \geq 0$ whenever $p_{i m b}(S) \leq u \leq P^{+}(S)$. Therefore any orbit $\left(S_{\varepsilon}, u_{\varepsilon}\right)$ will be monotone in both components as long as it remains in $H^{-}$, and the function $w_{\varepsilon}$ introduced above is well defined. 


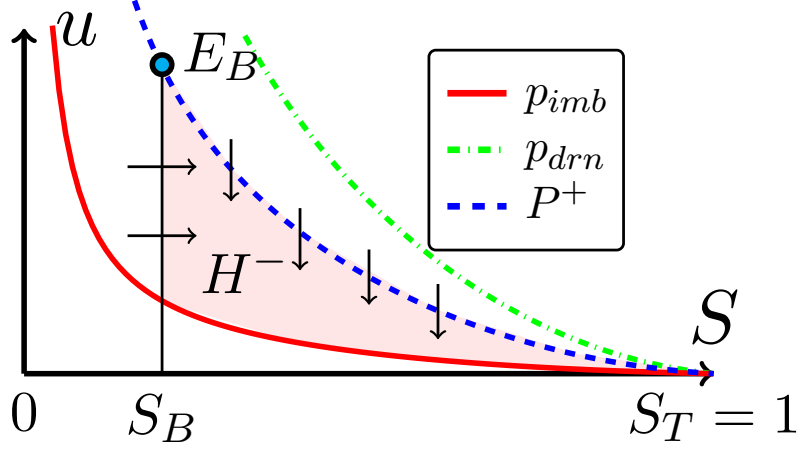

Figure 3: The invariant set $H^{-}$in the $S$ - $u$ plane. The arrows indicate direction of orbits with $\zeta$ increasing.
Referring to Figure 3, since $S_{\varepsilon}^{\prime}=0$ and $u_{\varepsilon}^{\prime}<0$ along the graph of $P^{+}$, the orbit cannot leave $H^{-}$through the upper boundary. The same holds for the vertical boundary $S=S_{B}$, since along it one has $S_{\varepsilon}^{\prime}>0$. Finally, as the orbit approaches the primary imbibition curve $p_{i m b}$ one has $S_{\varepsilon}^{\prime} \rightarrow+\infty$ and therefore $\frac{d w_{\varepsilon}}{d S} \rightarrow 0$. Since $p_{i m b}^{\prime}<0$, this implies that the orbit cannot leave $H^{-}$through the lower boundary as well. Hence $H^{-}$is invariant.

The next proposition is characterising the equilibrium point $E_{B}$.

Proposition 3.3 $E_{B}$ is a saddle type equilibrium.

Proof Linearizing (3.2)-(3.3) around any equilibrium point $E_{\alpha}=\left(S_{\alpha}, P^{+}\left(S_{\alpha}\right)\right)(\alpha \in\{B, T\})$ yields the characteristic equation

$$
\lambda^{2}-\Phi_{\varepsilon}^{\prime}(0) \frac{P^{+^{\prime}}\left(S_{\alpha}\right)}{P^{-}\left(S_{\alpha}\right)} \lambda+\Phi_{\varepsilon}^{\prime}(0) \frac{\left(k^{\prime}\left(S_{\alpha}\right)-c\right)}{k\left(S_{\alpha}\right) P^{-}\left(S_{\alpha}\right)}=0 .
$$

Since $k$ is convex one has $k^{\prime}\left(S_{B}\right)<c<k^{\prime}(1)$. For $E_{B}$, by Proposition 3.1 and Assumption (A.3), the last term on the right is negative, which proves the proposition.

Remark 3.1 Since $\Phi_{\varepsilon}^{\prime}(0)=\varepsilon$, one can immediately determine the asymptotic behaviour of the positive eigenvalue in (3.6) for the equilibrium point $E_{B}$. Specifically, with $C_{1,2}>0$ properly chosen one has

$$
\lambda_{+, B, \varepsilon}=\sqrt{C_{1}^{2} \varepsilon^{2}+C_{2} \varepsilon}-C_{1} \varepsilon=\mathcal{O}(\sqrt{\varepsilon}), \quad \text { as } \varepsilon \rightarrow 0 .
$$

Now we can prove Theorem 3.1.

Proof $(a)$ With given $\varepsilon>0$, we consider the unique orbit $\left(S_{\varepsilon}, u_{\varepsilon}\right)$ of the dynamical system (3.2)-(3.3) that leaves the saddle $E_{B}$ along the unstable direction corresponding to $\lambda_{+, B, \varepsilon}$ in Remark 3.1, and for increasing $S_{\varepsilon}$. By direct calculation one obtains that the corresponding eigenvector points into the region $H^{-}$. By Proposition 3.2, the orbit $\left(S_{\varepsilon}, u_{\varepsilon}\right)$ remains in $H^{-}$and therefore it is monotone in both components. Furthermore, as $\zeta \rightarrow \infty$, since the orbit does not leave $H^{-}$and its monotonicity prevents it from returning to $E_{B}$ or approaching a limit cycle, the orbit approaches the second equilibrium $E_{T}=(1,0)$. In other words, we have proved the first point in Theorem 3.1.

(b) For the second point we observe that the monotone behaviour of the orbit $\left(S_{\varepsilon}, u_{\varepsilon}\right)$ allows using the function $w_{\varepsilon}$ introduced in Definition 3.1. Letting $S \rightarrow S_{B}$ in (3.5) gives

$$
w_{\varepsilon}^{\prime}\left(S_{B}\right)=\frac{P^{+^{\prime}}\left(S_{B}\right)}{2}\left(1+\sqrt{1+\frac{4\left(c-k^{\prime}\left(S_{B}\right)\right) P^{-}\left(S_{B}\right)}{k\left(S_{B}\right) \Phi_{\varepsilon}^{\prime}(0)\left(P^{+^{\prime}}\left(S_{B}\right)\right)^{2}}}\right) .
$$


Let now $\varepsilon_{2}>\varepsilon_{1}>0$. Since $\Phi_{\varepsilon}^{\prime}(0)=\varepsilon$, from (3.7) one obtains $w_{\varepsilon_{2}}^{\prime}\left(S_{B}\right)>w_{\varepsilon_{1}}^{\prime}\left(S_{B}\right)$. As $w_{\varepsilon_{2}}\left(S_{B}\right)=w_{\varepsilon_{1}}\left(S_{B}\right)=P^{+}\left(S_{B}\right)$, this implies that a $\delta>0$ exists s.t. $w_{\varepsilon_{2}}(S)>w_{\varepsilon_{1}}(S)$ for all $S \in\left(S_{B}, S_{B}+\delta\right)$. Assume that the two orbits intersect in the interior of $H^{-}$, i.e. a $S^{*} \in\left(S_{B}, 1\right)$ exists s.t. $w_{\varepsilon_{2}}\left(S^{*}\right)=w_{\varepsilon_{1}}\left(S^{*}\right)=u^{*}$. Then, for the left limits of the derivatives one has $w_{\varepsilon_{1}}^{\prime}\left(S^{*}-0\right) \geq w_{\varepsilon_{2}}^{\prime}\left(S^{*}-0\right)$. This gives a contradiction since at the point $\left(S^{*}, u^{*}\right) \in H^{-}$it holds that

$$
w_{\varepsilon_{2}}^{\prime}\left(S^{*}\right)=\frac{\mathcal{G}\left(S^{*} ; S_{B}, 1\right)}{\Phi_{\varepsilon_{2}}\left(\frac{P^{+}\left(S^{*}\right)-u^{*}}{P^{-}\left(S^{*}\right)}\right)}>\frac{\mathcal{G}\left(S^{*} ; S_{B}, 1\right)}{\Phi_{\varepsilon_{1}}\left(\frac{P^{+}\left(S^{*}\right)-u^{*}}{P^{-}\left(S^{*}\right)}\right)}=w_{\varepsilon_{1}}^{\prime}\left(S^{*}\right)
$$

(c) From the above we know that for any $S \in\left(S_{B}, 1\right), w_{\varepsilon}(S)$ decreases with $\varepsilon$ and is bounded from below by $p_{i m b}(S)$. This implies that along any sequence $\varepsilon \rightarrow 0$ the sequence $w_{\varepsilon}(S)$ has a limit. This allows defining the function

$$
\bar{w}:\left[S_{B}, 1\right] \rightarrow\left[0, P^{+}\left(S_{B}\right)\right], \quad \bar{w}(S)=\lim _{\varepsilon \rightarrow 0} w_{\varepsilon}(S) .
$$

Clearly, $\bar{w}(S) \geq p_{i m b}(S)$ for all $S$, and $\bar{w}$ is decreasing. Now suppose that a $S_{0} \in\left(S_{B}, 1\right)$ exists s.t. $\bar{w}\left(S_{0}\right)>p_{i m b}\left(S_{0}\right)$. Then, with $\delta>0$ small enough, $S_{0}-\delta>S_{B}$ and $w_{\varepsilon}(S)>\bar{w}(S)>$ $p_{i m b}\left(S_{0}-\delta\right)$ for all $S \in\left(S_{0}-\delta, S_{0}\right)$ and $\varepsilon>0$. Observe that all orbits are passing through the region (see Figure 4 )

$$
\mathcal{R}=\left\{(S, u): S_{0}-\delta / 2<S<S_{0} \text { and } p_{i m b}\left(S_{0}-\delta\right)<u<P^{+}(S)\right\} .
$$

Observe that on $\left[S_{0}-\frac{\delta}{2}, S_{0}\right]$ the function $\mathcal{G}$ is bounded from below away from $0,|\mathcal{G}| \geq C$ for some $C>0$. Defining

$$
m=\sup _{(S, u) \in \mathcal{R}}\left(\frac{P^{+}(S)-u}{P^{-}(S)}\right),
$$

we have $0<m<1$ since $\mathcal{R}$ does not touch the curve $p_{i m b}$. From (3.5) and due to the monotonicity of $\Phi_{\varepsilon}$, in $\mathcal{R}$ we have

$$
-w_{\varepsilon}^{\prime}(S) \geq \frac{C}{\Phi_{\varepsilon}(m)}
$$

Therefore by mean value theorem we get

$$
P^{+}\left(S_{B}\right)-P_{i m b}\left(S_{0}\right) \geq w_{\varepsilon}\left(S_{0}-\delta\right)-w_{\varepsilon}\left(S_{0}\right) \geq \frac{C \delta}{\Phi_{\varepsilon}(m)} .
$$

While the left hand side is bounded, the right hand side goes to $\infty$ as $\varepsilon \rightarrow 0$, leading to a contradiction. Therefore $\lim _{\varepsilon \rightarrow 0} w_{\varepsilon}(S)=p_{i m b}(S)$ and the convergence is uniform on any closed interval $\left[S_{B}+\mu, 1\right]$ with $\mu>0$. 


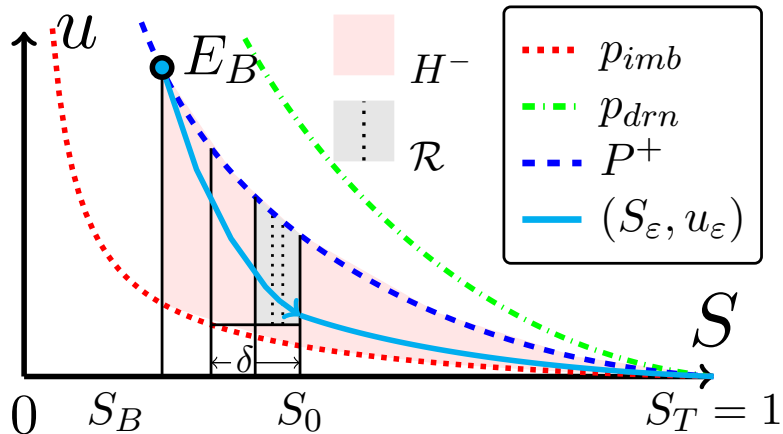

Figure 4: The saturation $S_{0}$ and the region $\mathcal{R}$ for $\varepsilon>0$.
Passing $\varepsilon \rightarrow 0$ gives TW solutions to the play-type hysteresis model. These are the limit of the orbits obtained in the regularised case, satisfying (2.20). We have

Corollary 3.1 Let $\zeta_{\varepsilon}^{*} \in \mathbb{R}$ be s.t. $u_{\varepsilon}(\zeta)=$ $p_{\text {imb }}\left(S_{B}\right)$ and let $S_{\varepsilon}^{*}=S_{\varepsilon}\left(\zeta_{\varepsilon}^{*}\right)$. Then

$$
\lim _{\varepsilon \rightarrow 0} S_{\varepsilon}^{*}=S_{B} \quad \text { and } \quad \lim _{\varepsilon \rightarrow 0} \zeta_{\varepsilon}^{*}=-\infty
$$

Before giving the proof we observe that in view of the convergence result in Theorem 3.1 (c), this corollary shows that for $\varepsilon \searrow 0$ the orbits are becoming vertical when approaching $E_{B}$.

Proof Since the orbits $\left(S_{\varepsilon}, u_{\varepsilon}\right)$ are ordered, $S_{\varepsilon}^{*}$ decreases with $\varepsilon$. Moreover, $S_{\varepsilon}^{*} \geq S_{B}$ since $w_{\varepsilon}\left(S_{B}\right)=P^{+}\left(S_{B}\right)$, so there exists $S^{*}=\lim _{\varepsilon \rightarrow 0} S_{\varepsilon}^{*}$ along any sequence $\varepsilon \rightarrow 0$. With this, the first result is obtained by contradiction, repeating the proof for point (c) of Theorem 3.1.

To show that $\zeta_{\varepsilon}^{*} \rightarrow-\infty$ as $\varepsilon \rightarrow 0$ we observe that the function $\eta_{\varepsilon}$ in Definition 3.1 is the inverse of $S_{\varepsilon}$. By (3.2), it satisfies

$$
\eta_{\varepsilon}^{\prime}=\frac{1}{\Phi_{\varepsilon}\left(\frac{P^{+}(S)-u}{P^{-}(S)}\right)}
$$

As $S_{\varepsilon}^{*}=S\left(\zeta_{\varepsilon}^{*}\right)$ and $S_{\varepsilon}(0)=\left(S_{B}+S_{T}\right) / 2$, integrating (3.10) and using (3.5) gives

$$
-\zeta_{\varepsilon}^{*}=\int_{S_{\varepsilon}^{*}}^{S_{\varepsilon}(0)} \frac{d S}{\Phi_{\varepsilon}}=\int_{S_{\varepsilon}^{*}}^{S_{\varepsilon}(0)} \frac{w_{\varepsilon}^{\prime}(S)}{\mathcal{G}\left(S ; S_{B}, 1\right)} d S .
$$

Since $S_{\varepsilon}^{*} \rightarrow S_{B}$, for any $\delta>0$ there exists a $\bar{\mu}=\bar{\mu}(\delta)$ s.t. $S_{B}<S_{\varepsilon}^{*}<S_{B}+\delta$ for all $0<\varepsilon<\bar{\mu}(\delta)$. As $\left.\mathcal{G} \in C^{1}\left(\left[S_{B}, 1\right]\right]\right)$, with $m=\max \left\{\left|\mathcal{G}^{\prime}(S)\right|, S \in\left[S_{B}, \frac{1}{2}\left(S_{T}+S_{B}\right)\right]\right\}$ it holds

$$
-\zeta_{\varepsilon}^{*} \geq \frac{1}{m} \int_{S_{\varepsilon}^{*}}^{S_{\varepsilon}(0)} \frac{-w_{\varepsilon}^{\prime}(S)}{S-S_{B}} d S \geq \frac{1}{m} \int_{S_{B}+\delta}^{\frac{1}{2}\left(S_{T}+S_{B}\right)} \frac{-w_{\varepsilon}^{\prime}(S)}{S-S_{B}} d S=: \frac{1}{m} h_{\varepsilon} .
$$

To evaluate $h_{\varepsilon}$, the last integral in the above, we use the uniform convergence of $w_{\varepsilon}$ to $p_{i m b}$. However, in $h_{\varepsilon}$ the derivative $w_{\varepsilon}^{\prime}$ appears, therefore we proceed as follows

$$
h_{\varepsilon}=\frac{w_{\varepsilon}\left(S_{B}+\delta\right)}{\delta}-\frac{2 w_{\varepsilon}\left(\frac{1}{2}\left(S_{T}+S_{B}\right)\right)}{\left(S_{T}-S_{B}\right)}-\int_{S_{B}+\delta}^{\frac{1}{2}\left(S_{T}+S_{B}\right)} \frac{w_{\varepsilon}(S)}{\left(S-S_{B}\right)^{2}} d S .
$$

The uniform convergence of $w_{\varepsilon}$ to $p_{i m b}$ proved in Theorem 3.1 gives

$$
\begin{aligned}
\lim _{\varepsilon \rightarrow 0} h_{\varepsilon}=h_{0} & =\frac{p_{i m b}\left(S_{B}+\delta\right)}{\delta}-\frac{2 p_{i m b}\left(\frac{1}{2}\left(S_{T}+S_{B}\right)\right)}{S_{T}-S_{B}}-\int_{S_{B}+\delta}^{\frac{1}{2}\left(S_{T}+S_{B}\right)} \frac{p_{i m b}(S)}{\left(S-S_{B}\right)^{2}} d S \\
& =\int_{S_{B}+\delta}^{\frac{1}{2}\left(S_{T}+S_{B}\right)} \frac{-p_{i m b}^{\prime}}{\left(S-S_{B}\right)} d S
\end{aligned}
$$

as $p_{i m b} \in \mathcal{C}^{1}$. Therefore a $\mu^{*}(\nu) \leq \bar{\mu}$ exists s.t. $h_{\varepsilon}>h_{0}-\nu$ for all $\varepsilon \in\left(0, \mu^{*}(\nu)\right)$. 
Using this and (3.11), and observing that Assumption (A.3) guarantees the existence of a $m_{p}>0$ s.t. $-m_{p} \leq p_{i m b}^{\prime}(S)$ for $S_{B} \leq S \leq 1$, if $0<\varepsilon<\mu^{*}(\nu)$ one has

$$
-\zeta_{\varepsilon}^{*} \geq \frac{1}{m} \int_{S_{B}+\delta}^{\frac{1}{2}\left(S_{T}+S_{B}\right)} \frac{-p_{i m b}^{\prime}}{\left(S-S_{B}\right)} d S-\frac{\nu}{m} \geq \frac{m_{p}}{m} \ln \left(\frac{S_{T}-S_{B}}{2 \delta}\right)-\frac{\nu}{m} .
$$

As $\delta$ was taken arbitrarily small, from this we can conclude that $\zeta_{\varepsilon}^{*} \rightarrow-\infty$ as $\varepsilon \rightarrow 0$.

\subsection{The case $S_{T}<1$}

We consider now the case when the top and bottom saturations satisfy $0<S_{B}<S_{T}<1$. In the analysis we use the region

$$
H=\left\{(S, u): S_{B} \leq S \leq 1, p_{i m b}(S) \leq u \leq p_{d r n}(S)\right\},
$$

and its subregions (see Figure 5)

$$
\begin{aligned}
& H_{1}=\left\{(S, u): S_{B} \leq S \leq S_{T}, P_{i m b}(S) \leq u \leq P^{+}(S)\right\} \\
& H_{2}=\left\{(S, u): S_{T} \leq S \leq 1, P_{i m b}(S) \leq u \leq P^{+}(S)\right\}, \\
& H_{3}=\left\{(S, u): S_{T} \leq S \leq 1, P^{+}(S) \leq u \leq P_{d r n}(S)\right\}, \\
& H_{4}=\left\{(S, u): S_{B} \leq S \leq S_{T}, P^{+}(S) \leq u \leq P_{d r n}(S)\right\} .
\end{aligned}
$$

We first analyse the case when $\varepsilon>0$.

\subsubsection{Properties for fixed $\varepsilon>0$}

The key properties of the orbits are stated in

Theorem 3.2 Let $0<S_{B}<S_{T}<1$ and $\varepsilon>0$. Then the following holds

(a) There exists a unique orbit $\left(S_{\varepsilon}, u_{\varepsilon}\right)$ satisfying $(3.2),(3.3),(2.20)$ and connecting $E_{B}$ and $E_{T}$.

(b) There exists a $\varepsilon_{m}>0$ s.t. $E_{T}$ is a stable spiral sink whenever $0<\varepsilon<\varepsilon_{m}$.
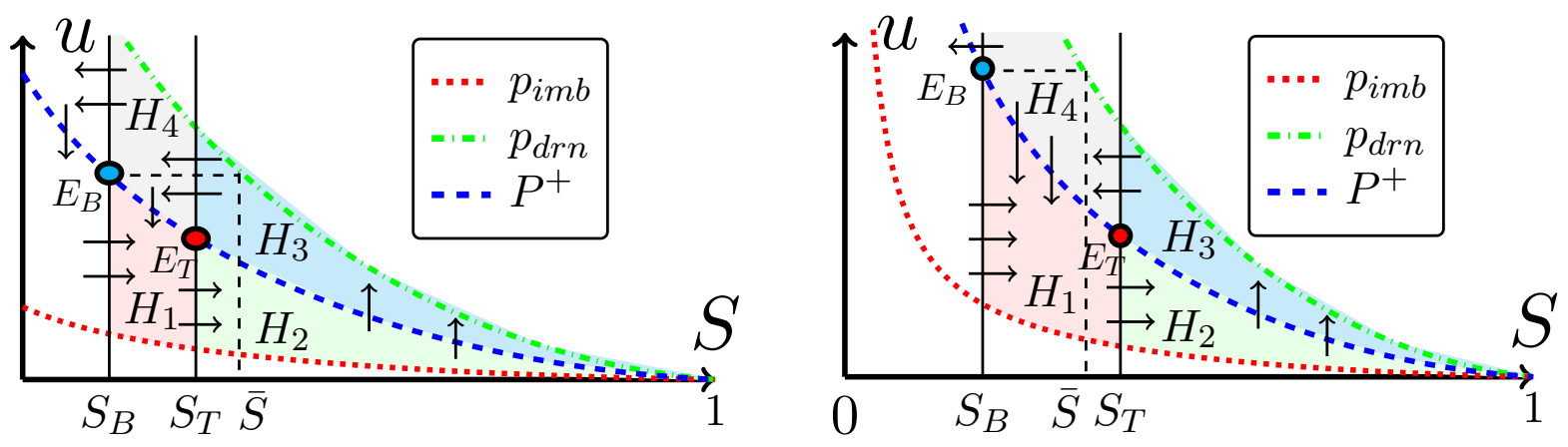

Figure 5: The regions $H_{1}, H_{2}, H_{3}$ and $H_{4}$ for the cases $S_{T}<\bar{S}$ (left) and $S_{T}>\bar{S}$ (right) where $\bar{S}$ is defined as $p_{d r n}(\bar{S})=P^{+}\left(S_{B}\right)$. The arrows indicate direction of orbits with $\zeta$ increasing. 
Proof (a) We observe that, repeating the proof for the $S_{T}=1$ case, the equilibrium $E_{B}$ is a saddle and all orbits leaving $E_{B}$ along the unstable direction and for increasing $S_{\varepsilon}$ enter the region $H^{-}$introduced in Proposition 3.2. Also, no orbit can leave the region $H$ defined above through the primary curves $p_{i m b}(S)$ and $p_{d r n}(S)$. Now we let $\bar{S}$ be s.t. $p_{d r n}(\bar{S})=P^{+}\left(S_{B}\right)$. Then two cases can be identified, $S_{T}<\bar{S}$ and $S_{T} \geq \bar{S}$.

The case $S_{T}<\bar{S}$ : As seen from the left picture in Figure 5, the orbit leaving $E_{B}$, for increasing $\overline{S_{\varepsilon}}$, enters first the region $H_{1}$. Then there are four possibilities (see Figure 5)

1. The orbit goes through $H_{2}, H_{3}, H_{4}$ and returns to $E_{B}$.

2. The orbit goes through $H_{2}, H_{3}, H_{4}$ and leaves $H_{4}$ through the segment $\left(S_{B}, p_{B}\right),\left(S_{B}, p_{d r n}\left(S_{B}\right)\right)$.

3. The orbit goes through $H_{2}, H_{3}, H_{4}$ and then leaves $H_{4}$ through the arc $\left(S, P^{+}(S)\right)$ between $E_{B}$ and $E_{T}$. This in turn gives rise to two possibilities:

A. The orbit moves around $E_{T}$ but does not approach it.

B. The orbit ends up in $E_{T}$.

The case $S_{T} \geq \bar{S}$ : In this case, if the orbit enters from $H_{3}$ to $H_{4}$ at some $\zeta=\zeta_{3-4}, u_{\varepsilon}\left(\zeta_{3-4}\right)<$ $\overline{p_{d r n}\left(S_{T}\right)<p_{d r n}(S)}=p_{B}$. But in $H_{4}, u_{\varepsilon}$ is decreasing, hence $u_{\varepsilon}<p_{B}$ for all arguments $\zeta>\zeta_{3-4}$, which rules out the first two possibilities (possibility 1 and 2) above.

To show that actually 3.B is the only possibility in both cases, we follow an argument from [17], based on Gauß Divergence Theorem. We define the vector-valued function

$$
\mathbf{F}: H \rightarrow \mathbb{R}^{2}, \quad \mathbf{F}(S, u)=\left(\Phi_{\varepsilon}(S, u), \mathcal{G}(S)\right),
$$

and denote its components by $\mathbf{F}_{S}$ and $\mathbf{F}_{u}$ respectively. A direct calculation gives

$$
\nabla \cdot \mathbf{F}=\frac{\Phi_{\varepsilon}^{\prime}}{\left(P^{-}\right)^{2}} \cdot\left(P^{+^{\prime}} P^{-}-P^{+} P^{-^{\prime}}+u{P^{-{ }^{\prime}}}^{\prime},\right.
$$

where the arguments $S$ and $u$ are disregarded. Hence, for $(S, u) \in H$ one has

$$
\nabla \cdot \mathbf{F}(S, u)<\left\{\begin{array}{l}
\frac{\Phi_{\varepsilon}^{\prime}}{\left(P^{-}\right)^{2}} \cdot\left(P^{+^{\prime}} P^{-}-P^{+} P^{-^{\prime}}+{P^{-{ }^{\prime}}} p_{i m b}\right) \text { if } P^{-^{\prime}}(S)<0 \\
\frac{\Phi_{\varepsilon}^{\prime}}{\left(P^{-}\right)^{2}} \cdot\left(P^{+^{\prime}} P^{-}-P^{+}{P^{-\prime}}^{-}+P^{-^{\prime}} p_{d r n}\right) \text { if } P^{-^{\prime}}(S)>0
\end{array}\right.
$$

The last factor in the first inequality gives

$$
\begin{aligned}
& \frac{1}{4}\left(p_{d r n}+p_{i m b}\right)^{\prime}\left(p_{d r n}-p_{i m b}\right)-\frac{1}{4}\left(p_{d r n}+p_{i m b}\right)\left(p_{d r n}-p_{i m b}\right)^{\prime}+\frac{p_{i m b}}{2}\left(p_{d r n}-p_{i m b}\right)^{\prime} \\
& =\frac{1}{4}\left(2 p_{d r n} p_{i m b}^{\prime}-2 p_{i m b} p_{i m b}^{\prime}\right)=\frac{1}{2}\left(p_{d r n}-p_{i m b}\right) p_{i m b}^{\prime}<0
\end{aligned}
$$

Similarly, in the second inequality one gets $\frac{1}{2}\left(p_{d r n}-p_{i m b}\right) p_{d r n}^{\prime}<0$. Thus we have shown that

$$
\nabla \cdot \mathbf{F}(S, u)<0 \quad \text { for all }(S, u) \in H .
$$

We can now investigate the possibilities mentioned above. To rule out the first two possibilities in the case $S_{T}<\bar{S}$ we define the domain $\Omega$ bounded by the closed orbit, or by the orbit and the segment $\left(S_{B}, p_{B}\right),\left(S_{B}, p_{d r n}\left(S_{B}\right)\right)$ (see Figure 6$)$. Let the orbit intersect the segment $\left(S_{B}, p_{B}\right)$, $\left(S_{B}, p_{d r n}\left(S_{B}\right)\right)$ at the point $T$. So for possibility $1, T$ is simply $E_{T}$. By (3.14) one has

$$
0>\int_{\Omega} \nabla \cdot \mathbf{F}=\int_{E_{B}}^{T} \mathbf{F} \cdot \hat{n}+\int_{T}^{E_{B}} \mathbf{F} \cdot \hat{n}=0-\int_{T}^{E_{B}} \mathbf{F}_{S},
$$



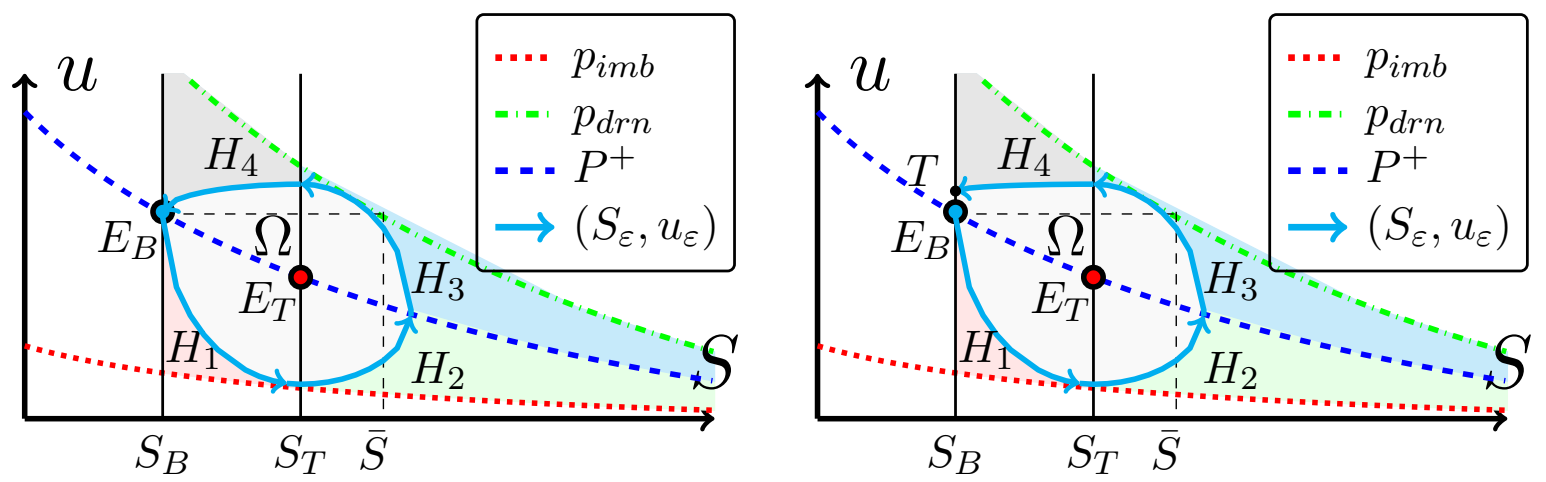

Figure 6: Possibility 1 (left) : orbit returning to $E_{B}$ after going through regions $H_{1}, H_{2}, H_{3}$ and $H_{4}$. Possibility 2 (right) : orbit exiting region $H_{4}$ through the segment $\left(S_{B}, p_{B}\right),\left(S_{B}, p_{d r n}\left(S_{B}\right)\right)$.

with the last integral on the right appearing only in the second possibility listed above. Since $\mathbf{F}_{S} \leq 0$ in the region $H \backslash H^{-}$, this gives a contradiction.

Finally, to eliminate 3.A we observe that by the Poincaré-Bendixson Theorem, if the orbit does not end up in $E_{T}$ then it must approach a limit cycle around $E_{T}$. However, one can use again the argument above, to show that limit cycles do not exist. So, the only possible behaviour of the orbits is as stated in possibility 3.B. This is displayed in the left plot of Figure 7 .

(b) Having proved the existence of an orbit connecting $E_{B}$ and $E_{T}$, showing that the orbit forms a stable spiral around $E_{T}$ for small enough $\varepsilon$ is a matter of calculation. Using the properties of $\Phi_{\varepsilon}, P^{+}$and the convexity of $k_{T}$ in (3.6) it is easy to show that for small values of $\varepsilon$, the eigenvalues corresponding to equilibrium point $E_{T}$ will be complex with negative real part. This completes the proof.

The left plot in Figure 7 shows the phase portrait in the $S$ - $u$ plane. In the right plot one has orbit component $S$ as function of $-\zeta$, in the case when $E_{T}$ is a stable spiral. Note the usage of $-\zeta=x-c t$ instead of $\zeta$, which is because in the original problem (with $x$ and $t$ as independent variables) the left state $(x=-\infty)$ corresponds to $S_{T}$ and right state $(x=\infty)$ corresponds to $S_{B}$. This convention will be useful when comparing with numerical solutions to (2.9)-(2.12).
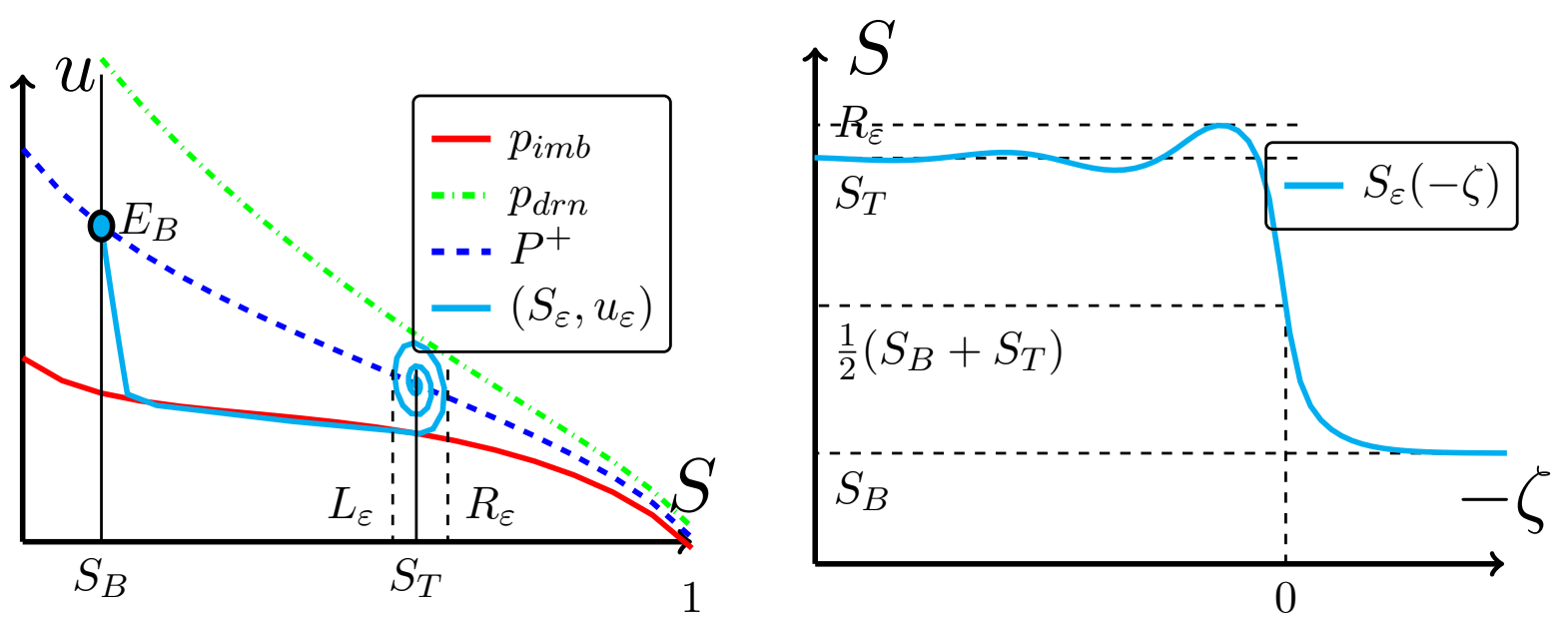

Figure 7: (left) Orbit connecting the saddle point to the spiral sink $E_{T}$, and (right) the profile of $S$ as a function of $-\zeta=x-c t$. 


\subsubsection{Properties for the limit case $\varepsilon \rightarrow 0$}

Knowing now the structure of the orbits for fixed $\varepsilon>0$, we study the limit behaviour $\varepsilon \rightarrow 0$. In certain aspects, the results obtained for $S_{T}=1$ and for $S_{T}<1$ are quite similar. The major difference is in the fact that the orbits are not monotone anymore. In consequence, the function $w_{\varepsilon}$ introduced in Definition 3.1 can only be defined as long as $S_{\varepsilon}$ remains monotone. Clearly, when starting from $E_{B}$ the monotonicity is lost for the first argument $\zeta$ where $S_{\varepsilon}(\zeta)=S_{T}$. We define $\zeta_{\varepsilon}^{T}$ as

$$
\zeta_{\varepsilon}^{T}=\min \left\{\zeta \in \mathbb{R} / S_{\varepsilon}(\zeta)=S_{T}\right\} .
$$

From now on we refer to the function $w_{\varepsilon}$ as the one obtained for $\zeta \in\left(-\infty, \zeta_{\varepsilon}^{T}\right]$. With this one has

Proposition 3.4 (a) For any $S \in\left(S_{B}, S_{T}\right), w_{\varepsilon}(S) \rightarrow p_{\text {imb }}(S)$ as $\varepsilon \rightarrow 0$. The convergence of $w_{\varepsilon}$ to $p_{i m b}$ is uniform on $\left[S_{B}+\delta, S_{T}\right]$ for any $\delta>0$.

(b) As long as $S \leq S_{T}$ the orbits $\left(S_{\varepsilon}, u_{\varepsilon}\right)$ are well ordered w.r.t $\varepsilon>0$, and do not intersect.

The proof is absolutely similar to the one for Theorem 3.1 and is skipped here.

For the case $S_{T}=1$, Corollary 3.1 is stating the limit behaviour of the orbits when $\varepsilon \searrow 0$. The type of the equilibrium $E_{B}$ remains unchanged in the case $S_{T}<1$ and a similar result can be proved in this case too. Specifically, if $\zeta_{\varepsilon}^{*} \in \mathbb{R}$ is s.t. $u_{\varepsilon}(\zeta)=p_{i m b}\left(S_{B}\right)$, for $S_{\varepsilon}^{*}=S_{\varepsilon}\left(\zeta_{\varepsilon}^{*}\right)$ one has

$$
\lim _{\varepsilon \rightarrow 0} S_{\varepsilon}^{*}=S_{B} \quad \text { and } \quad \lim _{\varepsilon \rightarrow 0} \zeta_{\varepsilon}^{*}=-\infty
$$

and the corresponding orbits become vertical when approaching $E_{B}$.

The situation changes for $E_{T}$ since the orbits $\left(S_{\varepsilon}, u_{\varepsilon}\right)$ become stable spirals. To understand this behaviour we let $\bar{\zeta}_{\varepsilon}=\min \left\{\zeta \in \mathbb{R} / S_{\varepsilon}(\zeta)=S_{T}\right\}$ and define

$$
R_{\varepsilon}=\sup \left\{S_{\varepsilon}(\zeta): u_{\varepsilon}(\zeta)=P^{+}\left(S_{\varepsilon}(\zeta)\right)\right\} \text { and } L_{\varepsilon}=\inf \left\{S_{\varepsilon}(\zeta): u_{\varepsilon}(\zeta)=P^{+}\left(S_{\varepsilon}(\zeta)\right), \zeta>\bar{\zeta}_{\varepsilon}\right\}
$$

and prove the following

Proposition 3.5 For $\bar{\zeta}_{\varepsilon}, L_{\varepsilon}$ and $R_{\varepsilon}$ introduced above, one has

$$
\lim _{\varepsilon \rightarrow 0} \bar{\zeta}_{\varepsilon}=\infty, \quad \lim _{\varepsilon \rightarrow 0} L_{\varepsilon}=S_{T} \quad \text { and } \quad \lim _{\varepsilon \rightarrow 0} R_{\varepsilon}=S_{T} .
$$

Proof The proof for $\bar{\zeta}_{\varepsilon}$ is almost identical to the proof of Corollary 3.1. For the remaining part we only consider $R_{\varepsilon}$, the proof for $L_{\varepsilon}$ being similar.

Clearly, $R_{\varepsilon} \geq S_{T}$. Assuming that a $\delta>0$ and a sequence $\varepsilon_{k} \rightarrow 0$ exists such that $R_{\varepsilon_{k}}>$ $S_{T}+\delta$ for all $k \in \mathbb{N}$. Let

$$
\mathcal{R}=\left\{(S, u): S_{T}+\frac{\delta}{2}<S<S_{T}+\delta \text { and } p_{i m b}\left(S_{T}\right) \leq u \leq P^{+}(S)\right\} .
$$

Clearly, all orbits pass through $\mathcal{R}$. Letting

$$
M=\sup _{(S, u) \in \mathcal{R}}\left(\frac{P^{+}(S)-u}{P^{-}(S)}\right)
$$

one has $0 \leq M<1$ and $0 \leq \Phi_{\varepsilon}\left(\frac{P^{+}(S)-u}{P^{-}(S)}\right)<\Phi_{\varepsilon}(M)$ for all $(S, u) \in \mathcal{R}$. From (3.5) and recalling that $k$ is convex, for any $S \in\left(S_{T}+\frac{\delta}{2},<S_{T}+\delta\right)$ one has

$$
w_{\varepsilon_{k}}^{\prime}(S)>\frac{\mathcal{G}\left(S ; S_{B}, S_{T}\right)}{\Phi_{\varepsilon_{k}}(M)}>\frac{\left(k(S)-k\left(S_{T}\right)\right)-c\left(S-S_{T}\right)}{k\left(S_{T}+\delta\right) \cdot \Phi_{\varepsilon_{k}}(M)} .
$$


Integrating the above over $\left(S_{T}+\frac{\delta}{2}, S_{T}+\delta\right)$ and using the properties of $k$, a constant $C_{0}>0$ depending on $\delta$ but not on $\varepsilon$ exists s.t.

$$
w_{\varepsilon_{k}}\left(S_{T}+\delta\right)-w_{\varepsilon_{k}}\left(S_{T}+\frac{\delta}{2}\right)>\frac{C_{0}}{\Phi_{\varepsilon_{k}}(M)} .
$$

In the above, the difference on the left is bounded by $P^{+}\left(S_{T}\right)-p_{i m b}\left(S_{T}+\delta\right)$. However, by Proposition 3.1, the ratio on the right goes to $\infty$ when $\varepsilon \rightarrow 0$, which gives a contradiction. This implies that $R_{\varepsilon} \rightarrow S_{T}$ for $\varepsilon \searrow 0$.
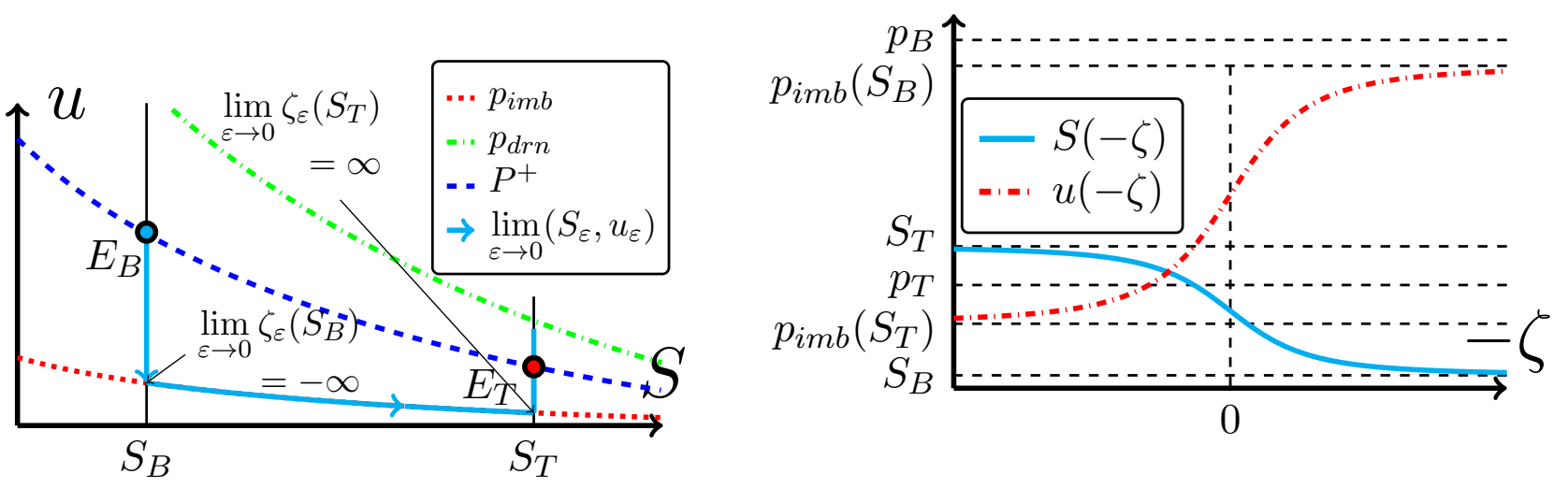

Figure 8: Orbit for limiting case $\varepsilon \rightarrow 0$ in $S$-u plane (left); and saturation and pressure profiles for the limiting orbit as a function of $-\zeta=x-c t$ (right).

Remark 3.2 Propositions 3.4 and 3.5 characterize the behaviour of the orbits in the limiting case $\varepsilon \rightarrow 0$. They are approaching vertical segments at $S=S_{B}$ and $S=S_{T}$, and in between the primary imbibition curve (see Figure 8). Possible oscillations can appear around $E_{T}$ when $S_{T}<1$. As $\varepsilon \rightarrow 0$, these oscillations are damped in the $S$ component, but we are unable to show similar behaviour for the pressure. Computational results shown in Figures ?? indicate that pressure oscillations do not decay as $\varepsilon$ decreases. However, these oscillations cannot be observed in reality for $\varepsilon \rightarrow 0$ as they are pushed towards infinity. Proceeding as in Corollary 3.1, one can show that $\zeta_{\varepsilon}^{*} \rightarrow-\infty$ as $\varepsilon \rightarrow 0$ and a similar result holds for the other side, determined by $S_{T}$. In another words, the oscillations move to $\infty$ and at any finite point the limiting waves are monotone in both saturation and pressure and they lie on the primary imbibition curve.

\section{Dynamic capillarity}

Now we discuss the case without hysteresis, but include dynamic effects in the $P_{c^{-}} S$ relationship. More precisely, we assume that the primary curves in (2.5) are the same, $p_{i m b}=p_{d r n}$, giving $P^{-}(S)=0$ and $P^{+}(S)=p_{i m b}(S)$ for all $S$. For the ease of presentation, as many results in this case are similar to the ones for the hysteresis model, we still use the notations $P^{ \pm}$.

At the same time we now take $\tau>0$ and thus (2.10) and (2.12) become $u=P^{+}(S)-$ $\tau f(S) \partial_{t} S$. With the TW velocity $c$ given in (2.18), the dynamical system (2.14)-(2.15) associated to the TW solutions become,

$$
\begin{aligned}
S^{\prime} & =\frac{P^{+}(S)-u}{c \tau f(S)}, \\
u^{\prime} & =\mathcal{G}\left(S ; S_{B}, S_{T}\right) .
\end{aligned}
$$


As before, we seek orbits that connect the equilibria $E_{B}=\left(S_{B}, P^{+}\left(S_{B}\right)\right)$ and $E_{T}=\left(S_{T}, P^{+}\left(S_{T}\right)\right)$, where $0<S_{B}<S_{T} \leq 1$. To fix the ideas we normalize the orbits by assuming that $S(0)=\left(S_{B}+S_{T}\right) / 2$. We mention that the analysis here completes the ones in $[17,44,47,48]$.

In the following analysis we distinguish the cases, $f \in L^{1}\left(S_{B}, 1\right)$ and $f \notin L^{1}\left(S_{B}, 1\right)$. In the former case one can define the primitive function $F:\left[S_{B}, 1\right] \rightarrow[0, \infty), F(S)=\int_{S_{B}}^{S} f(\varrho) d \varrho$. By Assumption (A.3) $F$ is one to one when its range is restricted to $[0, F(1)]$. With this, the transformation

$$
Y=F(S), \quad \text { impying } \quad S=F^{-1}(Y)
$$

is well defined and (4.1)-(4.2) becomes

$$
\begin{aligned}
Y^{\prime} & =\frac{P^{+}(S(Y))-u}{c \tau} \\
u^{\prime} & =\mathcal{G}\left(S(Y) ; S_{B}, S_{T}\right) .
\end{aligned}
$$

Observe that this system is similar to the one obtained for a constant damping function, $f \equiv 1$, as the functions $P^{+}(S(\cdot))$ and $\mathcal{G}(S(\cdot))$ have the same general properties as $P^{+}(\cdot)$ and $\mathcal{G}(\cdot)$. Hence we start analysing the existence of TW solutions and their properties by replacing (4.1) with the simpler equation

$$
S^{\prime}=\frac{P^{+}(S)-u}{c \tau},
$$

as the analysis for the system (4.6), (4.2) can be immediately transferred to the general case when $f$ is $L^{1}$. Moreover, the same applies for the case when $f \notin L^{1}$, as this is different from $f \in L^{1}$ only if $S_{T}=1$. However, the case $f \notin L^{1}$ gives a natural framework in which the saturation remains within the physically relevant range, $S \in[0,1]$.

\subsection{General behaviour of the orbits}

As for the hysteresis case, in this part we analyse the existence of orbits of the system (4.6), (4.2) connecting the equilibrium points $E_{B}$ and $E_{T}$. Clearly, these orbits will depend on $\tau$, motivating the notation $\left(S_{\tau}, u_{\tau}\right)$. Below we use the regions

$$
\begin{aligned}
& H_{1}=\left\{(S, u): S_{B} \leq S \leq S_{T}, u \leq P^{+}(S)\right\}, \quad H_{2}=\left\{(S, u): S_{T} \leq S \leq 1, u \leq P^{+}(S)\right\}, \\
& H_{3}=\left\{(S, u): S_{T} \leq S \leq 1, P^{+}(S) \leq u\right\}, \quad H_{4}=\left\{(S, u): S_{B} \leq S \leq S_{T}, P^{+}(S) \leq u\right\} .
\end{aligned}
$$

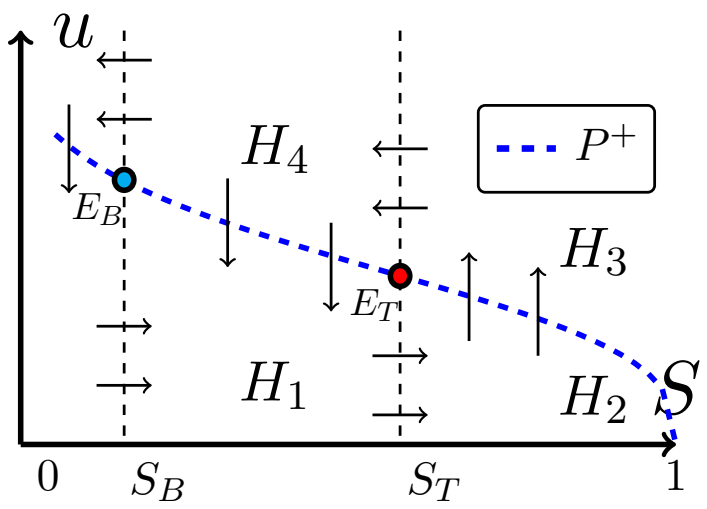

Figure 9: The directions followed by the orbits in the $S$ - $u$ plane for dynamic capillary case.
Figure 9 shows the directions followed by the orbits of the system (4.6), (4.2). Note that if an orbit goes through $H_{1}$, there it is monotone in both components, namely $u_{\tau}^{\prime}<0$ and $S_{\tau}^{\prime}>0$. Hence an orbit can only exit $H_{1}$ through the line $S=S_{T}$.

A straightforward calculation shows that the eigenvalues for the linearization of (4.6), (4.2) around $E_{\alpha}=\left(S_{\alpha}, P^{+}\left(S_{\alpha}\right)\right)(\alpha \in\{B, T\})$ are

$$
\lambda_{\tau}^{ \pm}=\frac{P^{\prime+}\left(S_{\alpha}\right)}{2 c \tau}\left(1 \pm \sqrt{1-\frac{4 c \tau\left(k^{\prime}\left(S_{\alpha}\right)-c\right)}{k\left(S_{\alpha}\right)\left(P^{+^{\prime}}\left(S_{\alpha}\right)\right)^{2}}}\right) .
$$


Since $k$ is convex, one has $k^{\prime}\left(S_{T}\right)>c>k^{\prime}\left(S_{B}\right)$, which shows that $E_{B}$ is a saddle point. Further, the unstable orbit leaving $E_{B}$ to the right enters the region $H_{1}$. To understand its behaviour as $\zeta \rightarrow \infty$ we begin with

Proposition 4.1 Given $\tau>0$, the orbit $\left(S_{\tau}, u_{\tau}\right)$ leaving $E_{B}$ into $H_{1}$ either approaches $E_{T}$ from $H_{1}$ as $\zeta \rightarrow \infty$, or leaves $H_{1}$ through vertical line $S=S_{T}$.

Proof In view of the monotonicity inside $H_{1}$, if $\left(S_{\tau}, u_{\tau}\right)$ does not leave $H_{1}$ through its right boundary it will approach an equilibrium contained in $H_{1}$ and at the right of $E_{B}$. Since $k$ is a convex function, the only such point is $E_{T}$.

As for the hysteresis model, all orbits $\left(S_{\tau}, u_{\tau}\right)$ are monotone between $\left(S_{B}, S_{T}\right)$. So, similar to Definition 3.1, with the stated normalization $S_{\tau}(0)=\left(S_{B}+S_{T}\right) / 2$ it is possible to define the functions $\eta_{\tau}, w_{\tau}:\left(S_{B}, S_{T}\right) \rightarrow \mathbb{R}$ for the dynamic capillarity model as well. More precisely, for any $S \in\left(S_{B}, S_{T}\right)$, a unique $\zeta_{w}$ exists s.t. $S_{\tau}\left(\zeta_{w}\right)=S$ and $S_{\tau}(\zeta)<S$ for all $\zeta<\zeta_{w}$. With this, $w_{\tau}(S)=u_{\tau}\left(\zeta_{w}\right)$ and $\eta_{\tau}(S)=\zeta_{w}$. Also one can extend $w_{\tau}$ to the closed interval $\left[S_{B}, S_{T}\right]$.

We emphasize on the fact that the functions are defined as long as $S_{\tau}$ remains increasing. In particular, this holds until the orbit leaves $H_{1} \cup H_{2}$. Similar to (3.5), $w_{\tau}$ satisfies the differential equation

$$
w_{\tau}^{\prime}(S)=\frac{\tau c \mathcal{G}\left(S ; S_{B}, S_{T}\right)}{P^{+}(S)-w_{\tau}} .
$$

The propositions below explain how the orbits $\left(S_{\tau}, u_{\tau}\right)$ depend on $\tau$, before they leave $H_{1}$.

Proposition 4.2 For the family of functions $w_{\tau}$ introduced above one has

(a) $w_{\tau} \rightarrow P^{+}$uniformly in $\left[S_{B}, S_{T}\right]$ as $\tau \rightarrow 0$.

(b) For any $S \in\left(S_{B}, S_{T}\right], w_{\tau}(S) \rightarrow-\infty$ as $\tau \rightarrow \infty$.

Proof We define the family of functions $v_{\tau}:\left[S_{B}, S_{T}\right] \rightarrow[0, \infty), v_{\tau}(S)=P^{+}(S)-w_{\tau}(S)$. Note that since $\left(S_{\tau}, u_{\tau}\right) \in H_{1}, v_{\tau}$ is always positive. By (4.8) we get

$$
\frac{1}{2}\left(v^{2}\right)^{\prime}(S)=v v^{\prime}(S)=-c \tau \mathcal{G}\left(S ; S_{B}, S_{T}\right)+v\left(P^{+}\right)^{\prime} \leq-c \tau \mathcal{G}\left(S ; S_{B}, S_{T}\right) .
$$

Integration from $S=S_{B}$ to an arbitrary $S \in\left(S_{B}, S_{T}\right)$ gives

$$
v^{2}(S) \leq-2 c \tau \int_{S_{B}}^{S} \mathcal{G}\left(\varrho ; S_{B}, S_{T}\right) d \varrho \leq-2 c \tau \int_{S_{B}}^{S_{T}} \mathcal{G}\left(\varrho ; S_{B}, S_{T}\right) d \varrho=2 \tau \bar{K},
$$

with $\bar{K}=-c \int_{S_{B}}^{S_{T}} \mathcal{G}(\varrho) d \varrho \geq 0$. This implies

$$
0 \leq P^{+}(S)-w_{\tau}(S) \leq \sqrt{2 \tau \bar{K}}
$$

Observing that $\bar{K}$ does not depend on $S$, the conclusion follows immediately.

For the second part, assume there exists $L>0$ and $S^{*} \in\left(S_{B}, S_{T}\right]$ s.t. $w_{\tau_{k}}\left(S^{*}\right)>P^{+}\left(S^{*}\right)-L$ for a sequence $\left\{\tau_{k}\right\}_{k \in \mathbb{N}}$ going to infinity. Since $w_{\tau_{k}}$ is strictly decreasing in $\left[S_{B}, S_{T}\right]$ we have $P^{+}(S)-w_{\tau_{k}}(S)<P^{+}(S)-P^{+}\left(S^{*}\right)+L$ if $S_{B}<S<S^{*}$. Since $\mathcal{G}\left(S ; S_{B}, S_{T}\right)<0$ in $H_{1}$ integration of (4.8) gives

$$
\begin{aligned}
w_{\tau_{k}}\left(S^{*}\right) & =w_{\tau_{k}}\left(S_{B}\right)+c \tau_{k} \int_{S_{B}}^{S^{*}} \frac{\mathcal{G}(\varrho)}{P^{+}(\varrho)-w_{\tau_{k}}(\varrho)} d \varrho \\
& <P^{+}\left(S_{B}\right)+c \tau_{k} \int_{S_{B}}^{S^{*}} \frac{\mathcal{G}\left(\varrho ; S_{B}, S_{T}\right)}{P^{+}(\varrho)-P^{+}\left(S^{*}\right)+L} d \varrho=P^{+}\left(S_{B}\right)-c \tau_{k} K_{s},
\end{aligned}
$$


with $K_{s}=\int_{S_{B}}^{S^{*}} \frac{\mathcal{G}\left(\varrho ; S_{B}, S_{T}\right)}{P^{+}(\varrho)-P^{+}\left(S^{*}\right)+L} d \varrho$. Clearly, $K_{s}>0$. Since $\lim _{k \rightarrow \infty} \tau_{k}=\infty$, this contradicts the assumed boundedness of $w_{\tau_{k}}$ and the proposition is proved.

The orbits depend continuously and monotonically on $\tau$, as follows from

Proposition 4.3 For all $S \in\left[S_{B}, S_{T}\right], w_{\tau}(S)$ is continuously decreasing w.r.t $\tau$.

Proof The proof for the monotonicity follows the arguments in the proof of Theorem 3.1, point (b) and is omitted.

For the continuity we take $S \in\left(S_{B}, S_{T}\right]$ and $0<\tau_{1}<\tau_{2}$, and use again the functions $v_{1}=P^{+}-w_{\tau_{1}}, 1 \in\{1,2\}$. From (4.9) and using the monotonicity of $w_{\tau}$ w.r.t. $\tau$ one obtains

$$
\frac{1}{2}\left(v_{2}^{2}-v_{1}^{2}\right)^{\prime}(S)=-c\left(\tau_{2}-\tau_{1}\right) \mathcal{G}\left(S ; S_{B}, S_{T}\right)+\left(v_{2}-v_{1}\right)(S)\left(P^{+}\right)^{\prime}(S)<-c\left(\tau_{2}-\tau_{1}\right) \mathcal{G}\left(S ; S_{B}, S_{T}\right) .
$$

With $\bar{K}$ defined above, integration gives

$$
0<v_{2}^{2}(S)-v_{1}^{2}(S)<2\left(\tau_{2}-\tau_{1}\right) \bar{K}
$$

which implies the continuity w.r.t. $\tau$ of $v$ and consequently of $w_{\tau}$.

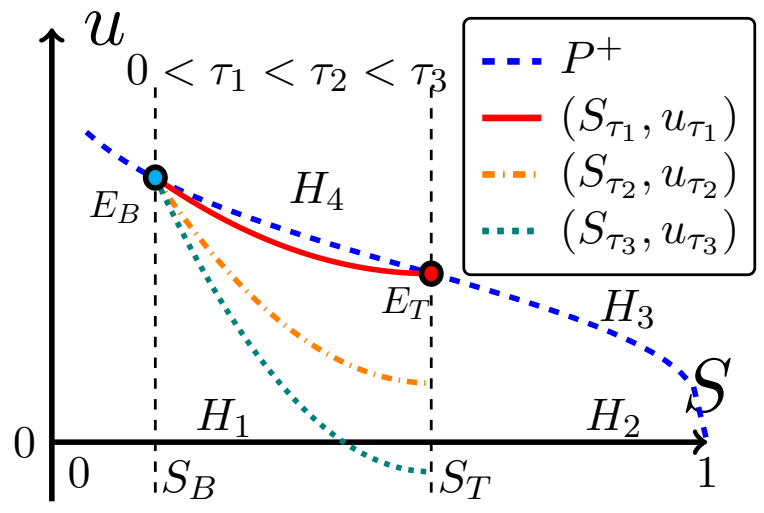

Figure 10: The dependency of the orbits $\left(S_{\tau}, u_{\tau}\right)$ on $\tau$ for $S_{B}<S<S_{T}$.
From the discussion so far we conclude that the orbits $\left(S_{\tau}, u_{\tau}\right)$ are close to the graph of $P^{+}$for small values of $\tau$, but move away from it as $\tau$ increases, and for $S \in\left(S_{B}, S_{T}\right]$. This situation is presented in Figure 10. In the remaining part of this subsection we focus on the behaviour of the system beyond the point $S=S_{T}$. The main goal is to show that orbits connecting $E_{B}$ and $E_{T}$ exist for all values of $\tau>0$. In Theorem 4.1 we show this for small values of $\tau$ and for larger $\tau$ values the existence is shown in Theorems 4.2 and 4.3.

Theorem 4.1 Let $\left\{\left(S_{\tau}, u_{\tau}\right)\right\}_{\tau>0}$ be the family of orbits of (4.6), (4.2), originating from $E_{B}$ and entering $H_{1}$. Then there exists a $\tau_{*}>0$ s.t. $w_{\tau_{*}}\left(S_{T}\right)=0$. For all $\tau \in\left(0, \tau_{*}\right]$ the system $(4.6)$, (4.2) has a unique orbit $\left(S_{\tau}, u_{\tau}\right)$ satisfying $S_{\tau}(0)=\left(S_{B}+S_{T}\right) / 2$ and connecting $E_{B}$ and $E_{T}$.

Proof The existence of a $\tau_{*}$ for which $w_{\tau_{*}}\left(S_{T}\right)=0$ follows directly from Propositions 4.2 and 4.3. Also, $w_{\tau}\left(S_{T}\right)<0$ for $\tau>\tau_{*}$ and $w_{\tau}\left(S_{T}\right)>0$ for $\tau<\tau_{*}$.

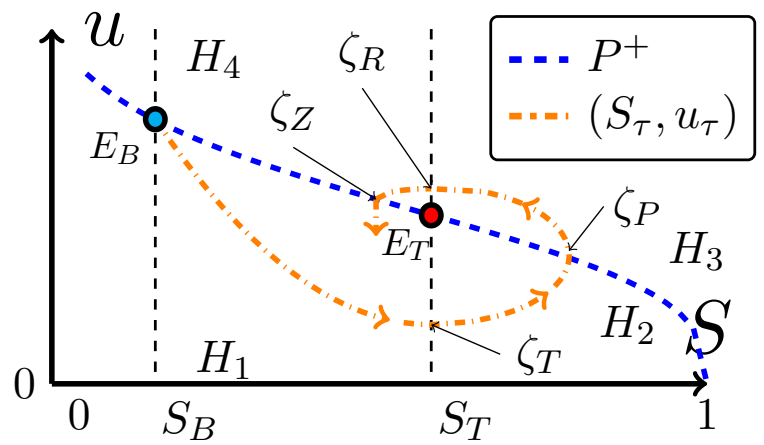

Figure 11: Behaviour of the orbit $\left(S_{\tau}, u_{\tau}\right)$ for $\tau \leq \tau_{*}$.
To understand the behaviour of $\left(S_{\tau}, u_{\tau}\right)$ for $\tau<\tau_{*}$ we recall Proposition 4.1, which states that the orbit either approaches $E_{T}$ or enters $H_{2}$ through $S=S_{T}$ at a finite $\zeta_{T}$. In the latter case, which is displayed in Figure 11, $u_{\tau}$ becomes increasing for $\zeta>\zeta_{T}$. With $\tau<$ $\tau_{*}$, since $P^{+}(1)=0<u_{\tau}\left(\zeta_{T}\right)<P^{+}\left(S_{T}\right)$ the orbit must intersect the graph of $P^{+}$at some $\zeta=\zeta_{P}$ and enter $H_{3}$, where $S_{\tau}$ becomes decreasing whereas $u_{\tau}$ is still increasing. We claim that the orbit either approaches $E_{T}$, or enters $H_{4}$ for some $\zeta=\zeta_{3}$. 
To see this, assume that a $\delta>0$ exists s.t. $S_{\tau}(\zeta) \geq S_{T}+\delta$ for all $\zeta>\zeta_{P}$. As $S_{\tau}$ is bounded and decreasing, the $\operatorname{limit}_{\lim _{\zeta \rightarrow \infty}} S_{\tau}(\zeta)$ exists and is finite. Denoting it by $\tilde{S}_{\tau}$ we have $\tilde{S}_{\tau} \geq S_{T}+\delta$. Further, since $u_{\tau}$ is only bounded from below, a similar reasoning shows that either $\lim _{\zeta \rightarrow \infty} u_{\tau}(\zeta)=\tilde{u}_{\tau} \in\left[P^{+}\left(\tilde{S}_{\tau}\right), \infty\right)$ or $u_{\tau} \rightarrow \infty$.

Since $S_{\tau}$ is decreasing with $\zeta$ and bounded from below, $\lim _{\zeta \rightarrow \infty} S_{\tau}^{\prime}=0$. From (4.6) one gets $\tilde{u}_{\tau}=P^{+}\left(\tilde{S}_{\tau}\right)$. Therefore $u_{\tau}$ has a (finite) limit as $\zeta \rightarrow \infty$ and from (4.2) we get $\lim _{\zeta \rightarrow \infty} u_{\tau}^{\prime}=0$. In other words, $\left(\tilde{S}_{\tau}, \tilde{u}_{\tau}\right)$ is an equilibrium point, which is not possible since $k$ is a convex function and therefore $\mathcal{G}$ has only two zeros. This rules out the possibility that $S_{\tau}$ is bounded away from $S_{T}$, so either $\lim _{\zeta \rightarrow \infty} S_{\tau}(\zeta)=S_{T}$, or the orbit enters $H_{4}$ at some finite argument $\zeta_{R}$.

In the former case it follows as before that the orbit ends up in $E_{T}$. In the latter case we follow the arguments in Theorem 3.2 to prove that $\left(S_{\tau}, u_{\tau}\right)$ cannot end up back in $E_{B}$, or leave $H_{4}$ through the line $S=S_{B}$. This means that it enters $H_{1}$ again at some $\zeta=\zeta_{Z}$. However, in this case the incoming part of the orbit is above the part emerging from $E_{B}$, and therefore the set bounded by $\left\{\left(S_{\tau}(\zeta), u_{\tau}(\zeta)\right) / \zeta<\zeta_{Z}\right\}$ and the graph of $P^{+}$from $E_{B}$ to $\left(S_{\tau}\left(\zeta_{Z}\right), u_{\tau}\left(\zeta_{Z}\right)\right)$ is positive invariant. With this, the proof continues as in Theorem 3.2.

Theorem 4.1 states that the orbits go to $E_{T}$ for all $\tau \in\left(0, \tau_{*}\right]$ but it does not state how the orbits behave close to $E_{T}$. This is given in

Proposition 4.4 There exists a $\tau_{m}>0$ s.t. for $\tau \in\left(0, \tau_{m}\right]$ any orbit going to $E_{T}$ goes either directly or after a finite number of turns around $E_{T}$, and for $\tau \in\left(\tau_{m}, \tau_{*}\right)$ the orbit is a stable spiral around $E_{T}$.

Proof To prove this part we use the eigenvalues of the linearization around $E_{T}$, computed in (4.7). Let $\tau_{m}=\frac{k\left(S_{T}\right)\left(P^{+^{\prime}}\left(S_{T}\right)\right)^{2}}{4 c\left(k^{\prime}\left(S_{T}\right)-c\right)}$. Note that $E_{T}$ is a stable sink for $0<\tau \leq \tau_{m}$ and a stable spiral for $\tau>\tau_{m}$. This proves the statement of Proposition 4.4.

Having explained the behaviour of orbits close to $E_{T}$ we again turn to existence, this time for $\tau>\tau_{*}$. As will be seen below, the orbits connecting $E_{B}$ and $E_{T}$ exist for $\tau>\tau_{*}$ too, but to prove this we have to introduce

$$
\alpha\left(S_{B}, S_{T}\right)=\int_{S_{B}}^{1} \mathcal{G}\left(\varrho ; S_{B}, S_{T}\right) d \varrho .
$$

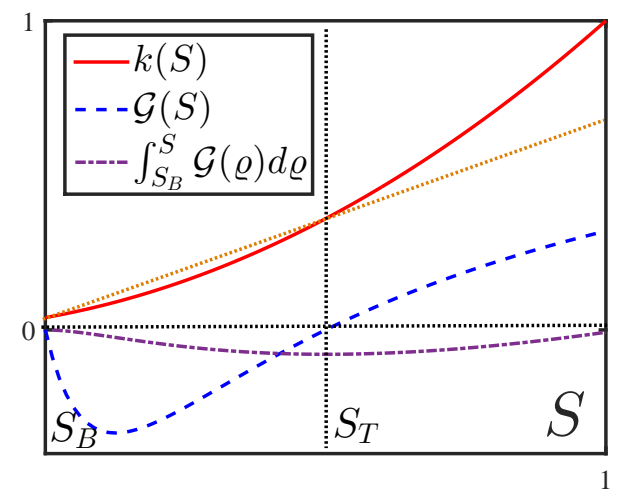

Figure 12: The functions $k, \mathcal{G}$, and the primitive of $\mathcal{G}$.
By the convexity of $k$, as stated in Assumption (A.1), and the definition $\mathcal{G}\left(S ; S_{B}, S_{B}\right)=$ $1-\frac{k^{\prime}\left(S_{B}\right)\left(S-S_{B}\right)+k\left(S_{B}\right)}{k(S)}$, for any fixed $S \in\left(S_{B}, 1\right]$ the function $\mathcal{G}$ is decreasing w.r.t. $S_{T} \in\left(S_{B}, 1\right]$. Also, one has $\mathcal{G}\left(S ; S_{B}, S_{T}\right)<0$ if $S \in\left(S_{B}, S_{T}\right)$ and $\mathcal{G}\left(S ; S_{B}, S_{T}\right)>0$ if $S \in\left(S_{T}, 1\right]$. Moreover,

$$
\alpha\left(S_{B}, S_{B}\right)>0>\alpha\left(S_{B}, 1\right)
$$

and $\alpha\left(S_{B}, \cdot\right)$ is decreasing in $\left[S_{B}, S_{T}\right]$. Observe that $\alpha\left(S_{B}, S_{T}\right)$ does not depend on $\tau$. Figure 12 shows how the functions $k(S), \mathcal{G}(S)$ and $\int_{S_{B}}^{S} \mathcal{G}\left(\varrho ; S_{B}, S_{T}\right) d \varrho$ vary with $S$.

With this we can now state the main result of this section,

Theorem 4.2 Let $S_{B}, S_{T} \in(0,1], S_{B}<S_{T}$ and $\alpha\left(S_{B}, S_{T}\right)$ be defined as above. If $\alpha\left(S_{B}, S_{T}\right) \geq$ 0 then for all $\tau>\tau_{*}$ the orbit $\left(S_{\tau}, u_{\tau}\right)$ reaches $E_{T}$ as $\zeta \rightarrow \infty$. 
Proof Since $\alpha\left(S_{B}, S_{T}\right) \geq 0$, by the properties of $\mathcal{G}$ an $S_{\alpha} \in\left[S_{T}, 1\right)$ exists s.t.

$$
\int_{S_{\alpha}}^{1} \mathcal{G}\left(S ; S_{B}, S_{T}\right) d S=\alpha\left(S_{B}, S_{T}\right)
$$

Clearly, $S_{\alpha}<1$ and $S_{\alpha}=1$ if $\alpha\left(S_{B}, S_{T}\right)=0$. We rewrite (4.8) as

$$
\frac{d}{d S}\left(P^{+}(S) w_{\tau}-\frac{1}{2} w_{\tau}^{2}\right)=c \tau \mathcal{G}(S)+w_{\tau} \frac{d P^{+}}{d S} .
$$

Since $\tau>\tau_{*}, w_{\tau}\left(S_{T}\right)<0$. Let $S_{1}(\tau) \in\left(S_{B}, S_{T}\right)$ be s.t. $w_{\tau}(S)>0$ for all $S \in\left[S_{B}, S_{1}(\tau)\right)$, i.e. the first point where the orbit $\left(S_{\tau}, u_{\tau}\right)$ enters the region $u<0$. Observe that $w_{\tau}$ is increasing for $S>S_{T}$. Further, let $S_{2}(\tau) \in\left(S_{T}, 1\right]$ be s.t. $w_{\tau}\left(S_{2}(\tau)\right)=0$ and $w_{\tau}(S)<0$ for all $S \in\left(S_{1}(\tau), S_{2}(\tau)\right)$. We prove that $S_{2}(\tau)<1$, thus the orbit returns in the upper half plane (see also Figure 13). More precisely, since $\alpha\left(S_{B}, S_{T}\right) \geq 0$, we prove in Proposition 4.6 that $S_{2}(\tau)<S_{\alpha}$ for all $\tau>\tau_{*}$.

Assume that $S_{2}(\tau)=1$ for some $\tau>\tau_{*}$, then the domain of definition of $w_{\tau}$ can be extended to $\left[S_{B}, 1\right]$. Integrating $(4.15)$ from $S_{1}(\tau)$ to 1 gives

$$
-\frac{1}{2} w_{\tau}^{2}(1)=c \tau \int_{S_{1}}^{1} \mathcal{G}+\int_{S_{1}}^{1} w_{\tau} \frac{d P^{+}}{d S} .
$$

Moreover, for $S \in\left(S_{1}(\tau), 1\right)$ one has $w_{\tau}(S)<0$ and since $\mathcal{G}\left(S ; S_{B}, S_{T}\right)<0$ for $S \in\left(S_{B}, S_{T}\right)$ one has

$$
\begin{aligned}
\alpha & =\int_{S_{B}}^{1} \mathcal{G}(S) d S=\int_{S_{B}}^{S_{1}} \mathcal{G}+\int_{S_{1}}^{1} \mathcal{G} \\
& =\int_{S_{B}}^{S_{1}} \mathcal{G}-\frac{1}{2 c \tau} w_{\tau}^{2}(1)-\frac{1}{c \tau} \int_{S_{1}}^{1} w_{\tau} \frac{d P^{+}}{d S}<0,
\end{aligned}
$$

which contradicts the assumption $\alpha\left(S_{B}, S_{T}\right) \geq 0$. Therefore, if $\tau>\tau_{*}$, a $S_{2}(\tau) \leq 1$ exists such that $w_{\tau}\left(S_{2}(\tau)\right)=0$, meaning that the orbit $\left(S_{\tau}, u_{\tau}\right)$ intersects the axis $u=0$ for the second time. Following the reasoning in the proof of Theorem 4.1 one obtains that $\left(S_{\tau}, u_{\tau}\right)$ ends up in $E_{T}$.

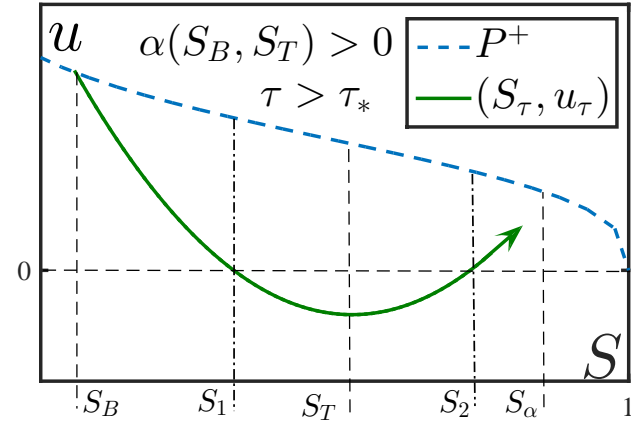

Figure 13: $\left(S_{\tau}, u_{\tau}\right)$ orbits for $\tau>\tau_{*}$ and $\alpha\left(S_{B}, S_{T}\right)>0 . S_{1}(\tau), S_{2}(\tau)$ and $S_{\alpha}$ are shown in the image for this particular $S_{T}$ value.
The proof of Theorem 4.2 introduces three important values for the saturation, $S_{\alpha}$ given by (4.14), and $S_{1}(\tau), S_{2}(\tau)$, the abscissas where the orbit intersects the axis $u=0$. Below we give some results on the boundedness of $w_{\tau}$ and of $S_{\alpha}$ and $S_{2}(\tau)$. We start with

Proposition 4.5 Let $\tau>\tau_{*}$ be such that $S_{2}(\tau) \in\left(S_{T}, 1\right]$ exists. Then

$$
w_{\tau}\left(S_{T}\right)>-\underline{K} \sqrt{\tau}
$$

where $\underline{K}^{2}=2 c \int_{S_{T}}^{1} \mathcal{G}(S) d S$

Proof Equation (4.8) gives $\left(P^{+}(S)-w_{\tau}\right) w_{\tau}^{\prime}=c \tau \mathcal{G}(S)$. As $w_{\tau}^{\prime}(S)>0$ for $S \in\left(S_{T}, S_{2}(\tau)\right]$, this gives $-w_{\tau}^{2^{\prime}}(S)<2 c \tau \mathcal{G}(S)$. The proof follows by intergating this inequality over $\left(S_{T}, S_{2}(\tau)\right]$. 
Observe that the estimate in Proposition 4.5 gives a lower bound for $w_{\tau}$ since $w_{\tau}\left(S_{T}\right)$ is a minimum for $w_{\tau}$. Also, the result does not require that $\alpha\left(S_{B}, S_{T}\right) \geq 0$.

The behaviour of $S_{\alpha}$ and $S_{2}(\tau)$ is stated in

Proposition 4.6 Under the assumptions of Theorem 4.2, one has $S_{2}(\tau)<S_{\alpha}$ and $\lim _{\tau \rightarrow \infty} S_{2}(\tau)=$ $S_{\alpha}$.

Proof To estimate $S_{2}(\tau)$ we integrate (4.15) from $S_{1}(\tau)$ to $S_{2}(\tau)$ and obtain

$$
c \tau \int_{S_{1}(\tau)}^{S_{2}(\tau)} \mathcal{G}(S)+\int_{S_{1}(\tau)}^{S_{2}(\tau)} w_{\tau} \frac{d P^{+}}{d S}=0
$$

Using this, one can split the integrals in (4.12) to obtain

$$
\int_{S_{2}(\tau)}^{1} \mathcal{G}=\alpha-\int_{S_{B}}^{S_{1}(\tau)} \mathcal{G}+\frac{1}{c \tau} \int_{S_{1}(\tau)}^{S_{2}(\tau)} w_{\tau} \frac{d P^{+}}{d S}
$$

Denoting by $I_{1}(\tau)$ and $I_{2}(\tau)$ the two integrals on the right, since $\mathcal{G}<0$ for $S \in\left(S_{B}, S_{T}\right)$ and $w_{\tau}(S)<0$ for $S \in\left(S_{1}(\tau), S_{2}(\tau)\right)$ one gets $I_{1}(\tau)<0$ and $I_{2}(\tau)>0$. This gives $\int_{S_{2}(\tau)}^{S_{\alpha}} \mathcal{G}>0$. As $S_{2}(\tau)>S_{T}, \mathcal{G}>0$ for $S \in\left(S_{2}(\tau), 1\right)$ and therefore $S_{2}(\tau)<S_{\alpha}$ for all $\tau>\tau_{*}$.

To obtain the limit we start by proving that $S_{1}(\tau) \rightarrow S_{B}$ as $\tau \rightarrow \infty$. Clearly $S_{1}(\tau)$ decreases with increasing $\tau$ and remains bounded from below by $S_{B}$. Now suppose $S_{1}(\tau) \geq S_{B}+\delta$ for some $\delta>0$ and for all $\tau>\tau_{*}$. Since $w_{\tau}(S)>0$ and $\mathcal{G}(S)<0$ for $S \in\left(S_{B}, S_{1}(\tau)\right)$, integrating (4.8) from $S_{B}$ to $S_{1}(\tau)$ gives

$$
P^{+}\left(S_{B}\right)=c \tau \int_{S_{B}}^{S_{1}} \frac{-\mathcal{G}(S)}{P^{+}(S)-w_{\tau}(S)} d S>-\frac{c \tau}{P^{+}\left(S_{B}\right)} \int_{S_{B}}^{S_{B}+\delta} \mathcal{G}(S) d S .
$$

This gives a contradiction for large $\tau$ as $c$ and $\mathcal{G}$ do not depend on $\tau$. Hence $\lim _{\tau \rightarrow \infty} I_{1}(\tau)=0$.

To estimate $I_{2}$ we use Proposition 4.5 and the properties of $w_{\tau}$

$$
0<I_{2}(\tau)=\frac{1}{c \tau} \int_{S_{1}}^{S_{2}} u_{\tau} \frac{d P^{+}}{d S}<\frac{1}{c \sqrt{\tau}} P^{+}\left(S_{B}\right) \underline{K} .
$$

Hence $\lim _{\tau \rightarrow \infty} \int_{S_{2}}^{1} \mathcal{G}(S) d S=\alpha=\int_{S_{\alpha}}^{1} \mathcal{G}(S) d S$. This proves that $S_{2} \rightarrow S_{\alpha}$ for $\tau \rightarrow \infty$.

Having understood the behaviour of the orbits for the case $\alpha\left(S_{B}, S_{T}\right) \geq 0$ we proceed by analysing the case $\alpha\left(S_{B}, S_{T}\right)<0$. We have

Lemma 4.1 Let $S_{B}, S_{T} \in(0,1], S_{B}<S_{T}$ and $\alpha\left(S_{B}, S_{T}\right)$ introduced in (4.12). If $\alpha\left(S_{B}, S_{T}\right)<$ 0 then $a \tau^{*}>\tau_{*}$ exists s.t. for all $\tau>\tau^{*}$, the orbit $\left(S_{\tau}, u_{\tau}\right)$ passes through a point $\left(1, w_{\tau}(1)\right)$ with $w_{\tau}(1)<0$.

Proof We use ideas that are similar to the ones in the proof of Theorem 4.2. Assume that $S_{2}(\tau) \leq 1$ for all $\tau>\tau_{*}$. Integrating (4.15) from $S=S_{B}$ to $S=S_{2}(\tau)$ gives

$$
\begin{aligned}
-\frac{1}{2} P^{+}\left(S_{B}\right)^{2} & =c \tau \int_{S_{B}}^{S_{2}(\tau)} \mathcal{G}(S)+\int_{S_{B}}^{S_{2}(\tau)} w_{\tau} \frac{d P^{+}}{d S}<c \tau \alpha+w_{\tau}\left(S_{T}\right)\left(P^{+}\left(S_{2}\right)-P^{+}\left(S_{B}\right)\right) \\
& <c \tau \alpha-w_{\tau}\left(S_{T}\right) P^{+}\left(S_{B}\right)<c \tau \alpha+P^{+}\left(S_{B}\right) \underline{K} \sqrt{\tau} .
\end{aligned}
$$

Since $\alpha<0$ this gives a contradiction for $\tau$ exceeding a $\tau^{*} \geq \tau_{*}$, where $\tau^{*}$ is determined s.t. the term on the right in the equation above becomes equal to $-\frac{1}{2} P^{+}\left(S_{B}\right)^{2}$. From this it follows that for $\tau>\tau^{*}$ the orbit $\left(S_{\tau}, u_{\tau}\right)$ has no second intersection point with the $u$-axis before passing through the vertical line $S=1$, therefore $w_{\tau}(1)<0$. 
From Lemma 4.1 we see that, if $\alpha\left(S_{B}, S_{T}\right)<0$ and $\tau$ is large enough, the orbit $\left(S_{\tau}, u_{\tau}\right)$ exits the domain $[0,1] \times \mathbb{R}$ through the halfline $\{(1, u), u<0\}$. Nevertheless, the functions $P^{+}$ and $k$ are only defined inside the physically relevant regime $S \in[0,1]$. One possibility is to extend the definition of $P^{+}$and $\mathcal{G}$ to $[0, \infty)$ e.g. by letting them become constant for $S \geq 1$. However, such values for $S$ are not physical and need to be avoided. To this aim we consider the multivalued extension

$$
P_{e}(S)= \begin{cases}P^{+}(S), & \text { for } 0<S<1 \\ (-\infty, 0] & \text { for } S=1 .\end{cases}
$$

Observe that its inverse is the capacity function $C: \mathbb{R} \rightarrow(0,1]$ (see e.g. [45]). Such an approach is also being used for defining extended pressure conditions in the case of porous media with block-type heterogeneities, and if models involving an entry pressure are adopted (see e.g. $[40,9])$. Further, for mathematical purpose $k$ is extended in such a way that $\mathcal{G}(S)=\mathcal{G}(1)>0$ for all $S \geq 1$. However, we prove below that the choice of $P_{e}$ guarantees that $S$ does not exceed 1. To do so we let $\delta>0$ be a small regularisation parameter and define

$$
P_{e}^{\delta}(S)=\left\{\begin{array}{l}
P^{+}(S), \quad \text { for } 0<S<1 \\
\frac{1}{\delta}(1-S) \quad \text { for } S \geq 1
\end{array}\right.
$$

With this, let $\left(S_{\tau}^{\delta}, u_{\tau}^{\delta}\right)$ be the orbit originating from $E_{B}$ and satisfying (4.6), (4.2) with $P^{+}(S)$ replaced by $P_{e}^{\delta}(S)$. We are interested in the limit behaviour when $\delta \searrow 0$, which is stated in

Theorem 4.3 With $\tau^{*}$ from Lemma 4.1, for any $\tau>\tau^{*}$ and $\delta>0$ the orbits $\left(S_{\tau}^{\delta}, u_{\tau}^{\delta}\right)$ emerging from $E_{B}$ end up in $E_{T}$. Furthermore, for any $\zeta \in \mathbb{R}$, the limit $\lim _{\delta \rightarrow 0}\left(S_{\tau}^{\delta}(\zeta), u_{\tau}^{\delta}(\zeta)\right)$ exists and it satisfies $\lim _{\delta \rightarrow 0} S_{\tau}^{\delta}(\zeta) \leq 1$.

Proof Let $\tau>\tau^{*}$ be fixed and $\zeta_{1}$ be s.t. $S_{\tau}(\zeta)<1$ for $\zeta<\zeta_{1}$ and $S_{\tau}\left(\zeta_{1}\right)=1$. Observe that, for all $\delta>0$, the orbits $\left(S_{\tau}^{\delta}, u_{\tau}^{\delta}\right)$ exist and coincide with $\left(S_{\tau}, u_{\tau}\right)$ if $\zeta \leq \zeta_{1}$. This gives trivially the existence of $\lim _{\delta \rightarrow 0}\left(S_{\tau}^{\delta}(\zeta), u_{\tau}^{\delta}(\zeta)\right)$ for $\zeta \leq \zeta_{1}$, and that $\zeta_{1}$ does not depend on $\delta$.

Now for $\delta>0$, since $u_{\tau}^{\delta}$ is increasing whenever $S_{\tau}^{\delta} \geq 1 \geq S_{T}$ whereas $P_{e}^{\delta}$ is decreasing for $S \geq 1$, the orbit $\left(S_{\tau}^{\delta}, u_{\tau}^{\delta}\right)$ will intersect the curve $u=P_{e}^{\delta}(S)$. Following now the ideas in the proof of Theorem 4.1 one obtains that the orbit ends up in $E_{T}$.

To analyse further the limit case we observe first that the orbits $\left(S_{\tau}^{\delta}, u_{\tau}^{\delta}\right)$ are well-ordered w.r.t. $\delta$ for $\zeta>\zeta_{1}$. To see this, as long as the saturation $S_{\tau}^{\delta}$ remains increasing we define $w_{\tau}^{\delta}$ similar to $w_{\tau}$ and observe that for $0<\delta_{1}<\delta_{2}$, (4.8) implies that $0<w_{\tau}^{\delta_{2}}(S)<w_{\tau}^{\delta_{1}}(S)$ for all $S>1$ where both $w$ functions are defined. Also, the two orbits cannot intersect. The behaviour of the orbits is shown in Figure 14. 


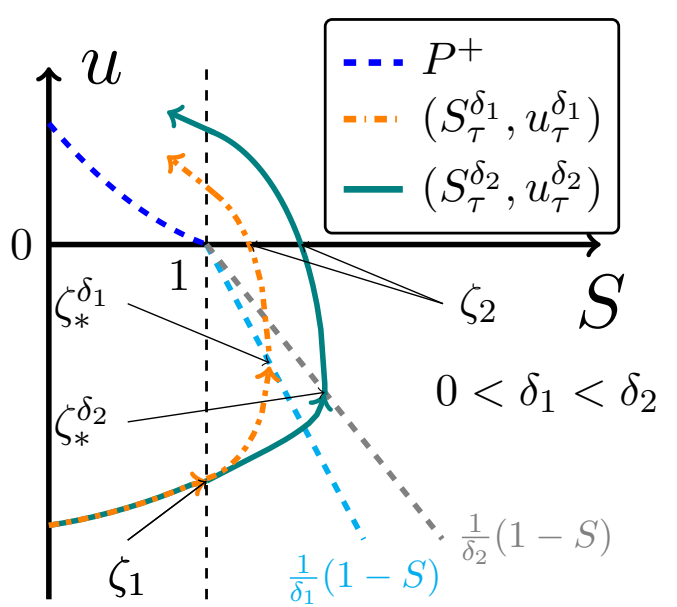

Figure 14: Behaviour of the orbits $\left(S_{\tau}^{\delta}, u_{\tau}^{\delta}\right)$ for different $\delta$ values.
As discussed above, for any $\delta>0$ the orbit intersects the graph of the regularised $P_{e}^{\delta}$ function. Let $\left(S_{*}^{\delta}, P_{e}^{\delta}\left(S_{*}^{\delta}\right)\right)$ be this intersection point and $\zeta_{*}^{\delta} \in \mathbb{R}$ the corresponding argument. Since $u_{\tau}^{\delta}$ becomes increasing for $\zeta>\zeta_{*}^{\delta}$, an argument $\zeta_{2}$ exists s.t. $u_{\tau}^{\delta}\left(\zeta_{2}\right)=0$. Note that since all orbits are identical until crossing the line $S=1$ whereas $\mathcal{G}$ is constant for $S>1$, from (4.2) it follows that $\zeta_{2}$ is constant w.r.t. $\delta$. From the definition of $P_{e}^{\delta}$ and (4.10) one gets

$$
P^{+}\left(S_{T}\right)-\sqrt{2 \tau \bar{K}}<w_{\tau}\left(S_{T}\right)<\frac{1}{\delta}\left(1-S_{*}^{\delta}\right),
$$

which implies that

$$
1<S_{*}^{\delta}<1+\delta\left(\sqrt{2 \tau \bar{K}}-P^{+}\left(S_{T}\right)\right) .
$$

This proves that $\lim _{\delta \rightarrow 0} S_{*}^{\delta}$ exists and is equal to 1 . Moreover, it also gives that $\lim _{\delta \rightarrow 0} S_{\tau}^{\delta}(\zeta)=$ 1 for all $\zeta \in\left(\zeta_{1}, \zeta_{2}\right]$ since for such $\zeta$ one has $1<S_{\tau}^{\delta}(\zeta) \leq S_{*}^{\delta}$. Also, for $\zeta_{1}<\zeta \leq \zeta_{2}$, one has $u_{\tau}^{\delta}(\zeta)=\mathcal{G}(1)\left(\zeta-\zeta_{1}\right) \in\left(w_{\tau}(1), 0\right]$, which is independent of $\delta$. Hence $\lim _{\delta \rightarrow 0} u_{\tau}^{\delta}(\zeta)$ also exists and lies in the interval $\left(w_{\tau}(1), 0\right]$.

Finally, since $\lim _{\delta \rightarrow 0} S_{\tau}^{\delta}(\zeta)=1$ for all $\zeta \in\left(\zeta_{1}, \zeta_{2}\right]$ whereas $u_{\tau}^{\delta}(\zeta)>P_{e}^{\delta}\left(S_{\tau}^{\delta}(\zeta)\right)$ for $\zeta \in\left(\zeta_{*}^{\delta}, \zeta_{2}\right]$, the limit orbit will lie inside $[0,1] \times \mathbb{R}$ for $\zeta \geq \zeta_{2}$. In this case $P_{e}^{\delta}(S)=P^{+}(S)$. Hence the limit $\delta \rightarrow 0$ will exist in this case too, which completes the proof.

Having understood the above we can now distinguish the following situations which are shown in Figure 15. If $\alpha>0$ the orbits stay away from $S=1$ and approach $E_{T}$ either directly or after spiraling (see Figure 15a). The situation is similar if $\alpha<0$ and $\tau<\tau^{*}$. Whenever $\alpha<0$ and $\tau>\tau^{*}$ then the orbit $\left(S_{\tau}, u_{\tau}\right)$ can be defined as

$$
\left(S_{\tau}, u_{\tau}\right)=\lim _{\delta \rightarrow 0}\left(S_{\tau}^{\delta}, u_{\tau}^{\delta}\right)
$$

for all $\zeta \in \mathbb{R}$. These orbits have a vertical section at $S=1$. The orbits $\left(S_{\tau}, u_{\tau}\right)$ for $\alpha<0$ are shown in Figure 15b.

We conclude this part by observing that although the results are stated for $f(S)=1$, the coordinate transform in (4.3) allows extending these for the case of any positive function $f \in L^{1}\left(\left[S_{B}, 1\right]\right)$.

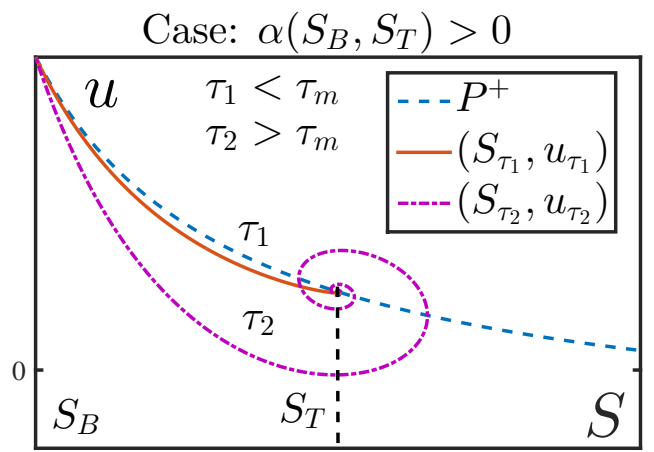

(a) Typical orbits for $\alpha\left(S_{B}, S_{T}\right)>0$

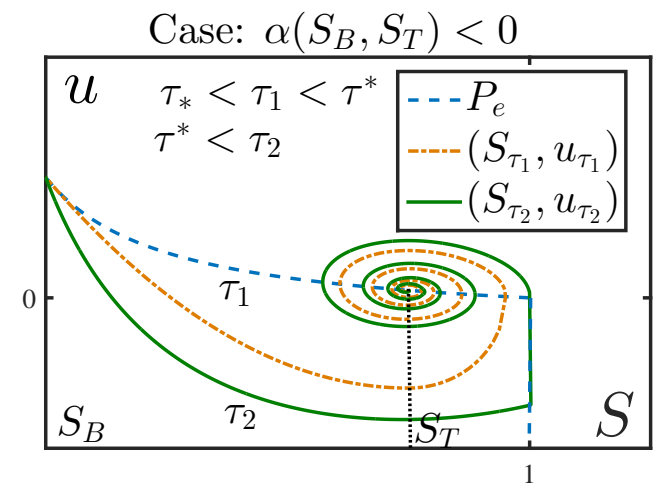

(b) Typical orbits for $\alpha\left(S_{B}, S_{T}\right)<0$

Figure 15: Behaviour of the orbits $\left(S_{\tau}, u_{\tau}\right)$ for $\tau>0$ and $f(S)=1$. 


\subsection{The case when $f \notin L^{1}\left(S_{B}, 1\right)$}

The TW analysis of the dynamic capillarity model up to now is restricted to the case when $f \in L^{1}\left(S_{B}, 1\right)$, namely $\int_{S_{B}}^{1} f(S) d S<\infty$. This might not always be true. Since $f$ is assumed continuous on $(0,1)$ and positive, $f \notin L^{1}\left(S_{B}, 1\right)$ implies that it may become unbounded at $S=0$ or $S=1$. In this case, two major issues are encountered. First, the arguments where $f$ becomes unbounded will become lower or upper bounds for the saturation. As above, if boundedness is violated at both $S=0$ and $S=1$, the orbits remain inside the physically relevant regime $0 \leq S \leq 1$ and extending the capillary pressure is not needed. Second, if $f$ becomes unbounded one can also incorporate the concept of residual saturation in the model. In general, the residual saturation gives the amount of one phase that can not be driven out by the infiltration of another phase (see e.g. [31]). The subsequent analysis will reveal how to incorporate the residual saturation in the travelling wave formulation.

Let $\delta>0$ be arbitrarily small. Whenever $S \leq 1-\delta$, one can apply the transformation (4.3) to reduce the model (4.1)-(4.2) to the case analysed in Subsection 4.1 and most of the results there still remaind valid. In particular, the orbits remain monotone if $S \in\left(S_{B}, S_{T}\right)$. The main difference appears close to $S=1$, if this value is approached. We have

Theorem 4.4 Assume $f \notin L^{1}\left(S_{B}, 1\right)$ and let $\tau>0, S_{B} \in(0,1), S_{T} \in\left(S_{B}, 1\right]$ be given. For the orbits $\left(S_{\tau}, u_{\tau}\right)$ leaving $E_{B}$ one has

(a) If $S_{T}<1$, then $S_{\tau}(\zeta)<1$ for all $\zeta \in \mathbb{R}$.

(b) If $S_{T}=1$, then two cases can occur.

(b.1) If $f \mathcal{G} \notin L^{1}\left(S_{B}, 1\right)$ then as $\zeta \rightarrow \infty, S_{\tau} \rightarrow 1$ and $u_{\tau} \rightarrow-\infty$.

(b.2) If $f \mathcal{G} \in L^{1}\left(S_{B}, 1\right)$ then there exists a $u^{*} \in\left(-\infty, P^{+}(1)\right]$ s.t. $\lim _{\zeta \rightarrow \infty}\left(S_{\tau}, u_{\tau}\right) \rightarrow\left(1, u^{*}\right)$.

Proof (a) Assume first that $S_{T}<1$. Compared to the situation analysed in Theorem 4.1, the differences appear whenever $S_{\tau}$ approaches 1 . We therefore focus on part of the orbit satisfying $S_{\tau}>S_{T}$. In this case, $u_{\tau}^{\prime}>0$ whereas $S_{\tau}^{\prime}>0$ as long as the orbit $\left(S_{\tau}, u_{\tau}\right)$ stays below the $P^{+}$ curve. Two situations are possible: the orbit either intersects the $P^{+}$curve for some argument $\zeta_{3}$, or it reaches the line $S=1$.

In the former situation, let $S_{3, \tau}=S_{\tau}\left(\zeta_{3}\right)$. We know that $S_{\tau}(\zeta) \leq S_{3}(\tau)$ for all $\zeta \in \mathbb{R}$, so if $S_{3, \tau}<1$ then the proof is completed. Assuming the contrary, namely that a $\tau_{0}>0$ exists such that $S_{3, \tau_{0}}=1$, one has $u_{\tau_{0}}\left(\zeta_{3}\right) \leq P^{+}(1)$ and (4.8) gives

$$
\frac{d w_{\tau}}{d S}(S)=\frac{\tau c f(S) \mathcal{G}(S)}{P^{+}(S)-w_{\tau}}
$$

As $P^{+^{\prime}}(S)<0$ and $\mathcal{G}(S)>0$ for $S \in\left[S_{T}, 1\right)$ one uses (4.10) to see that $P^{+}-w_{\tau_{0}} \geq 0$ decreases for $S \in\left[S_{T}, 1\right]$. Further, integration of (4.9) gives (with redefinition $\bar{K}=-c \int_{S_{B}}^{S_{T}} f(\varrho) \mathcal{G}(\varrho) d \varrho$ )

$$
\begin{aligned}
\sqrt{2 \tau_{0} \bar{K}} & >P^{+}\left(S_{T}\right)-w_{\tau_{0}}\left(S_{T}\right)>w_{\tau_{0}}(1)-w_{\tau_{0}}\left(S_{T}\right) \\
& =\int_{S_{T}}^{1} \frac{\tau_{0} c f(S) \mathcal{G}(S)}{P^{+}(S)-w_{\tau_{0}}(S)} d S \geq \frac{\tau_{0} c}{P^{+}\left(S_{T}\right)-w_{\tau_{0}}\left(S_{T}\right)} \int_{S_{T}}^{1} f(S) \mathcal{G}(S) d S \\
& \geq \frac{\tau_{0} c}{P^{+}\left(S_{T}\right)-w_{\tau_{0}}\left(S_{T}\right)} \int_{\frac{S_{T}+1}{2}}^{1} f(S) \mathcal{G}(S) d S \geq \frac{c \tau_{0} m_{\mathcal{G}}}{P^{+}\left(S_{T}\right)-w_{\tau_{0}}\left(S_{T}\right)} \int_{\frac{S_{T}+1}{2}}^{1} f(S) d S,
\end{aligned}
$$

with $m_{\mathcal{G}}=\min \left\{\mathcal{G}(S), \frac{1}{2}\left(S_{T}+S_{\beta}\right) \leq S \leq 1\right\}$. Since $m_{\mathcal{G}}>0$ and $f \notin L^{1}\left(S_{B}, 1\right)$, the integral on the right is unbounded, which gives a contradiction. 
The second case, when the orbit reaches the line $S=1$, can be ruled out by similar arguments. We omit the details here.

(b) For $S_{T}=1$, observe that $S_{\tau}^{\prime}(\zeta)>0$ for $\left(S_{\tau}, u_{\tau}\right) \in H_{1}$ and $S_{\tau}$ is bounded above by 1 following the arguments used for proving Corollary 3.1. Consequently $S_{\tau}$ has a limit $S_{\infty}$ for $\zeta \rightarrow \infty$. Assume $S_{\infty}<1$. We know that $u_{\tau}^{\prime}(\zeta)$ decreases monotonically for $\zeta \in \mathbb{R}$ so that there are two possibilities. If $\lim _{\zeta \rightarrow \infty} u_{\tau}(\zeta)=u_{\infty}>-\infty$ then from (4.1) and (4.2) it follows that $S_{\tau}^{\prime}$ and $u_{\tau}^{\prime}$ both have a limit as $\zeta \rightarrow \infty$. Moreover, since $S_{\tau}$ and $u_{\tau}$ have horizontal asymptotes, it means that $\lim _{\zeta \rightarrow \infty} S_{\tau}^{\prime}(\zeta)=\lim _{\zeta \rightarrow \infty} u_{\tau}^{\prime}(\zeta)=0$. From (4.2) we then get $\mathcal{G}\left(S_{\infty}\right)=0$, contradicting $S_{\infty}<1$. On the contrary, if $\lim _{\zeta \rightarrow \infty} u_{\tau}(\zeta)=-\infty$ then from $(4.2)$ we get

$$
S_{\tau}^{\prime}(\zeta)=\frac{P^{+}(S)-u_{\tau}(\zeta)}{c \tau f(S)} \geq \frac{P^{+}(S)}{c \tau f(S)} \geq \inf _{S \in\left[S_{B}, S_{\infty}\right]}\left\{\frac{P^{+}(S)}{c \tau f(S)}\right\}>0
$$

for all $\zeta>M_{\zeta}$ with some large enough $M_{\zeta}$. This means that $S_{\tau}$ cannot have a limit $S_{\infty}<1$. Therefore the only possibility remaining is $\lim _{\zeta \rightarrow \infty} S_{\tau}=S_{\infty}=1$.

Now let us consider the case $f \mathcal{G} \notin L^{1}\left(S_{B}, 1\right)$. Observe that since $\mathcal{G}<0$ for $S \in\left(S_{B}, 1\right)$ one has $\int_{S_{B}}^{1} f(-\mathcal{G})=\infty$. If $w_{\tau}$ tends to $u^{*}>-\infty$ then integrating (4.20) from $S_{B}$ to 1 and multiplying by -1 we get

$$
P^{+}\left(S_{B}\right)-u^{*}=\int_{S_{B}}^{1} \frac{-\tau c f(S) \mathcal{G}(S)}{P^{+}(S)-w_{\tau}(S)} d S>\frac{\tau c}{P^{+}\left(S_{B}\right)-u^{*}} \int_{S_{B}}^{1} f(S)(-\mathcal{G}(S)) d S,
$$

which is a contradiction since the term on the left is bounded whereas the integral on the right is not. Hence $\lim _{S \rightarrow 1} w_{\tau}=-\infty$.

Next, for $f \mathcal{G} \in L^{1}\left(S_{B}, 1\right)$ after redefining $\bar{K}$ as $\bar{K}=-c \int_{S_{B}}^{1} \mathcal{G} f$, Proposition 4.2 gives a lower bound for $w_{\tau}\left(S_{T}\right)$ that is uniform for all $S_{B}<S_{T} \leq 1$. Also observe that for a fixed $S_{B}, w_{\tau}\left(S ; S_{B}, S_{T}\right)$ are well ordered w.r.t. $S_{T}$ meaning that for $S_{B}<S_{T, 1}<S_{T, 2}<1$, $w_{\tau}\left(S ; S_{B}, S_{T, 1}\right)>w_{\tau}\left(S ; S_{B}, S_{T, 2}\right)$ in their common domain of definition. To see why this holds observe that for $S \in\left(S_{B}, S_{T, 1}\right)$ and $u<P^{+}(S)$,

$$
\frac{\tau c f(S) \mathcal{G}\left(S ; S_{B}, S_{T, 1}\right)}{P^{+}(S)-u}>\frac{\tau c f(S) \mathcal{G}\left(S ; S_{B}, S_{T, 2}\right)}{P^{+}(S)-u}
$$

with $\mathcal{G}\left(S ; S_{B}, S_{T, 1}\right)>\mathcal{G}\left(S ; S_{B}, S_{T, 2}\right)$ following from the convexity of $k$. Using (4.20) and proceeding as in the proof of Theorem 3.1 we conclude that the orbits are well-ordered in $S \in\left(S_{B}, S_{T, 1}\right)$ w.r.t. $S_{T}$. As $w_{\tau}\left(S ; S_{B}, S_{T, 1}\right)>w_{\tau}\left(S_{T, 1} ; S_{B}, S_{T, 1}\right)$ for $S>S_{T, 1}$, the well ordering holds throughout the common domain of definition. In view of the boundedness of $w_{\tau}\left(S_{T}\right)$ mentioned before, $\lim _{S_{T} \rightarrow 1} w_{\tau}\left(S_{T} ; S_{B}, S_{T}\right)=u^{*}>-\infty$. Finally proceeding like proof of Corollary 3.1 one proves that this value can be only attained as $\zeta \rightarrow \infty$. 


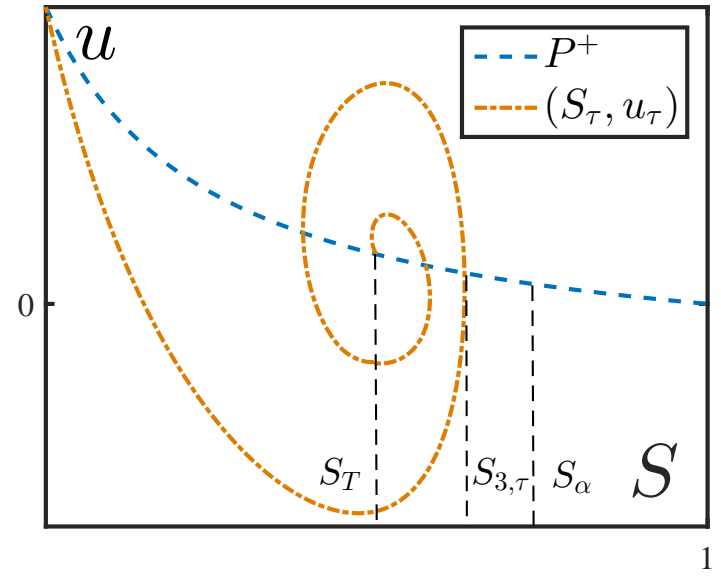

Figure 16: Typical $\left(S_{\tau}, u_{\tau}\right)$ orbit for the case $\int_{S_{B}}^{1} f(S) d S=\infty, S_{T}<1$.
From the proof above we see that in the case $S_{T}<1$, for any $\tau>0$ the orbit may turn around the equilibrium $E_{T}$ without reaching the line $S=1$. In particular, an $S_{3, \tau} \in\left(S_{T}, 1\right)$ exists s.t. the orbit interescts the graph of $P^{+}$for the first time after $E_{B}$ in the point $\left(S_{3, \tau}, P^{+}\left(S_{3, \tau}\right)\right)$, see Figure 16. Moreover, since $f \in C(0,1) \backslash L^{1}\left(S_{B}, 1\right)$ whereas $\mathcal{G} \in C[0,1]$ with $\mathcal{G}(1)>0$, one has $\lim _{S \nearrow_{1}} \int_{S_{B}}^{1} f(z) \mathcal{G}(z) d z=\infty$. Since $\mathcal{G}<0$ on $\left(S_{B}, S_{T}\right)$, a unique $S_{\alpha} \in\left(S_{T}, 1\right)$ exists s.t.

$$
\int_{S_{B}}^{S_{\alpha}} f(S) \mathcal{G}(S) d S=0
$$

Observe that this simply extends the definition of $S_{\alpha}$ in (4.12), given for the case $f \equiv 1$ to $f \in L^{1}\left(S_{B}, 1\right)$ and $f \notin L^{1}\left(S_{B}, 1\right)$. Having introduced the above, as in the case $f \equiv 1$, it is interesting to see what happens if $\tau$ becomes very large. We have:

Corollary 4.1 Let $S_{T}<1$ and $S_{\alpha}, S_{3, \tau}$ be as introduced above. Then $\lim _{\tau \rightarrow \infty} S_{3, \tau}=S_{\alpha}$.

Proof As in the proof of Proposition 4.3, the orbits are ordered w.r.t. $\tau$. Therefore $S_{3, \tau}$ is increasing w.r.t. $\tau$ and bounded from above, $S_{3, \tau}<1$. Hence there exists the limit $\lim _{\tau \rightarrow \infty} S_{3, \tau}=S_{3}^{*}$. As in Theorem 4.2, for $\tau>\tau_{*}$ and $S>S_{T}$ let $S_{2, \tau}$ be abscissa where the orbit intersects the axis $u=0$. Following the argument in Theorem 4.2, one proves that $\lim _{\tau \rightarrow \infty} S_{2, \tau}=S_{\alpha}$. Also as $S_{3, \tau}>S_{2, \tau}$ it is easy to see that $S_{3}^{*} \geq S_{\alpha}$. Now integrating (4.20) from $S_{2, \tau}$ to $S_{3, \tau}$ gives

$$
P^{+}\left(S_{T}\right)>w_{\tau}\left(S_{3, \tau}\right)=\int_{S_{2, \tau}}^{S_{3, \tau}} \frac{\tau c f(S) \mathcal{G}(S)}{P^{+}(S)-w_{\tau}(S)} d S \geq \frac{\tau c}{P^{+}\left(S_{T}\right)} \int_{S_{2, \tau}}^{S_{3, \tau}} f(S) \mathcal{G}(S) d S .
$$

Observe that if $S_{3}^{*}>S_{\alpha}$, since $\lim _{\tau \rightarrow \infty} S_{3, \tau}=S_{3}^{*}$ the integral on the right becomes positive for $\tau$ large enough. On the other hand, since the term on the left is bounded, as $\tau \rightarrow \infty$ this integral must approach 0 . For $S_{3}^{*}>S_{\alpha}$ this gives a contradiction, so the only possibility is $S_{3}^{*}=S_{\alpha}$.

Remark 4.1 For $S_{T}<1$, Corollary 4.1 shows that for all $\tau>0$ the orbits remain at the left of $S=S_{\alpha}<1$. This means that the travelling waves exist without needing to extend the capillary pressure in the non-physical domain $S>1$.

Remark 4.2 Observe that $\tau$ and $f$ have different effects. Specifically, changing $\tau$ affects the orbit for all values of $\zeta$, whereas $f$ plays a major role only in the vicinity of $S=1$.

Remark 4.3 The analysis above remains unchanged if $f$ has a singularity at some $S_{\beta} \in(0,1)$ s.t. $\int_{0}^{S_{\beta}} f(S) d s=\infty$. In this case the orbits will remain bounded at the right of the line $S=S_{\beta}$ for all $\tau>0$. So $S_{\beta}$ can be interpreted as a maximal saturation of the wetting phase (water), respectively $1-S_{\beta}$ is the residual saturation for the non-wetting phase (air). 


\section{$5 \quad$ Numerical Study}

The numerical results presented in this section complement the theoretical findings in the previous sections. Specifically, after solving numerically the system (2.9), (2.12) we verify the predictions made in previous sections for sufficiently large times. We start by presenting the numerical scheme.

\subsection{Numerical Scheme}

Below $\varepsilon>0$ and $\tau \geq 0$ are fixed. With $S_{B}<S_{T}$ we consider the system given by (2.9),(2.12) for $t>0$ and $x \in(-\ell, \ell)$. For the numerical solution we first discretize in time (2.9) and (2.12). Let $\Delta t>0$ be the time step and let $t_{n}=n \Delta t$ for $n \in \mathbb{N}$. The time discrete unknowns $S_{n}$, $u_{n}$ approximate the saturation and pressure at $t_{n}$. We introduce the function $\mathcal{F}$ which gives the discretization of $\partial_{t} S$. One gets from (2.12) that $\mathcal{F}(S, u)=c \Phi_{\varepsilon}\left(\frac{P^{+}(S)-u}{P^{-}(S)}\right)$ for the hysteresis case and $\mathcal{F}(S, u)=\frac{1}{c \tau}\left(P_{e}^{\delta}(S)-u\right)$ for the dynamic capillarity case. With the $\mathcal{F}$-notation, the explicit discretisation of $(2.12)$ reads

$$
S_{n}=S_{n-1}+\Delta t \mathcal{F}\left(S_{n-1}, u_{n-1}\right) .
$$

For stability we solve the time discrete version of (2.9) implicitly:

$$
\partial_{x}\left(k\left(S_{n}\right) \partial_{x} u_{n}\right)=-\mathcal{F}\left(S_{n}, u_{n}\right)-\partial_{x} k\left(S_{n}\right) .
$$

For (5.2) we use pressure boundary conditions at $x= \pm \ell$ :

$$
u_{n}(-\ell)=p_{T}, \quad u_{n}(\ell)=p_{B} .
$$

The initial condition is a smooth approximation of the Riemann data and it is consistent with the boundary conditions. Specifically $S_{0}:[-\ell, \ell] \rightarrow(0,1)$ satisfies

$$
S_{0}(x)=S_{T} \text {, if } x \leq-\ell_{1}, \quad \text { respectively } S_{0}(x)=S_{B} \text { if } x \geq \ell_{1} .
$$

Here $\ell_{1}<<\ell$ is a positive number, and $S_{T}$ and $S_{B}$ are compatible with the corresponding pressure values, i.e. $p_{\alpha}=P^{+}\left(S_{\alpha}\right)(\alpha \in\{B, T\})$.

For the dynamic capillary case, equation (5.2) is linear in $u_{n}$, but for the capillary hysteresis case it becomes non-linear and requires more attention. Observe that in this case the situation $\left|\mathcal{F}^{\prime}(S, u)\right| \rightarrow \infty$ arises in two different ways. Firstly, if $u \rightarrow p_{i m b}(S)$ or $u \rightarrow p_{d r n}(S)$, then $\mathcal{F}^{\prime}(S, u) \rightarrow \pm \infty$. To resolve this we define $\Phi_{\varepsilon}$ on $\mathbb{R}$ in a way such that $\Phi_{\varepsilon}^{\prime}(r)=\frac{1}{\gamma \varepsilon}$ for $|r|>1$ for some constant $\gamma>0$. Note that $\Phi_{\varepsilon}$ is different from the function $\Phi_{\varepsilon}$ given in Proposition 3.1, but satisfies Assumption (A.4)-(A.5). This particular choice guarantees the numerical convergence of the non-linear problem (5.2).

Secondly, $\left|\mathcal{F}^{\prime}(S, u)\right| \rightarrow \infty$ if $S \rightarrow 0$ or $S \rightarrow 1$. This problem is avoided by taking $S_{B}>0$ and $S_{T}<1$. So when studying the case $S_{T}=1$, we actually show the result for the limit $S_{T} \nearrow 1$. With these modifications, $\mathcal{F}$ becomes locally Lipschitz in both variables $S$ and $u$.

Because $\Phi_{\varepsilon}^{\prime}$ becomes unbounded as $\varepsilon \rightarrow 0$, iterative schemes like Newton's method fail to converge because of the requirement of having good initial guesses. Therefore to solve (5.2) we use a linear iteration scheme inspired by the L-scheme discussed in [36, 38]. Specifically, for a sufficiently large $L$ that will be specified later and with $i$ as the iteration index, we solve the linear elliptic equation

$$
L u_{n}^{i}-\partial_{x}\left(k\left(S_{n}\right) \partial_{x} u_{n}^{i}\right)=L u_{n}^{i-1}+\mathcal{F}\left(S_{n}, u_{n}^{i-1}\right)+\partial_{x} k\left(S_{n}\right)
$$


Following the arguments from [36, 38] one can show the existence of a constant $L_{\text {min }}>0$ s.t. for all $L>L_{\min }$ the scheme becomes a contraction and converges irrespective of the initial guess. In fact, $L_{\min }$ is the Lipschitz constant of $\mathcal{F}$ with respect to the variable $u$. A natural choice for the initial guess is $u_{n}^{0}=u_{n-1}$.

Observe that, for the hysteresis case, $L>\frac{1}{\gamma \varepsilon}$ guarantees the convergence of the iterations. However, with this choice the convergence is very slow, [36]. Observing that in most parts of the interval $(-\ell, \ell), S$ and $u$ are s.t. $\Phi_{\varepsilon}^{\prime}=\mathcal{O}(\varepsilon)$, one can improve this convergence by choosing an $x$-dependent $L$. Specifically, taking $L=\mathcal{L}:(-\ell, \ell) \rightarrow \mathbb{R}$ depending on $\Phi_{\varepsilon}^{\prime}$ restricted to a local sub-interval improves the local convergence of the iterations. In our computation we have chosen $L:=\mathcal{L}(x)=2 \mathcal{F}^{\prime}\left(S_{n-1}(x), u_{n-1}(x)\right)$ in every control volume.

\section{$5.2 \quad$ Numerical results}

As the problem is 1-D we use finite differences to discretise (5.2) and (5.5) in space. We use simple capillary pressure and permeability functions,

$$
P^{+}(S)=\left(\frac{1-S}{S}\right), \quad P^{-}(S)=2(1-S)^{2}, \quad k(S)=S^{2} .
$$

With $b=\sqrt[3]{\frac{\varepsilon}{\gamma}}$ and $a=\left(1-\left(\varepsilon^{2} \gamma\right)^{\frac{2}{3}}\right)$, the $\Phi_{\varepsilon}$ function used in the numerical scheme is

$$
\Phi_{\varepsilon}(r)= \begin{cases}b+\frac{1}{\gamma \varepsilon}(r-1) & \text { for } r>1 \\ \varepsilon r\left(1-a r^{2}\right)^{-1 / 2} & \text { for } r \in[-1,1] . \\ -b+\frac{1}{\gamma \varepsilon}(r+1) & \text { for } r<-1\end{cases}
$$

The interval is taken large enough to allow the saturation and pressure to develop profiles resembling the travelling wave ones and in all cases $\ell \geq 50$. We take a $C^{1}$ initial condition $S_{0}:[\ell, \ell] \rightarrow \mathbb{R}$ that approximates the jump from $S_{T}$ to $S_{B}$. Specifically, with $\ell_{1}=5$, we take $S_{0}(x)=\frac{\left(S_{B}+S_{T}\right)}{2}+\frac{\left(S_{T}-S_{B}\right)}{4 \ell_{1}^{3}} x\left(x^{2}-3 \ell_{1}^{2}\right)$ for $x \in\left[-\ell_{1}, \ell_{1}\right], S_{0}(x)=S_{T}$ for $x<-\ell_{1}$, and $S_{0}(x)=S_{B}$ for $x>\ell_{1}$. However it is to be noted that the choice of $\Phi_{\varepsilon}$ and $S_{0}$ do not have considerable impact on the end results as long as necessary assumptions are satisfied.

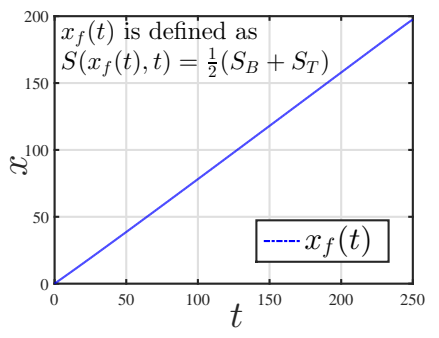

(a)

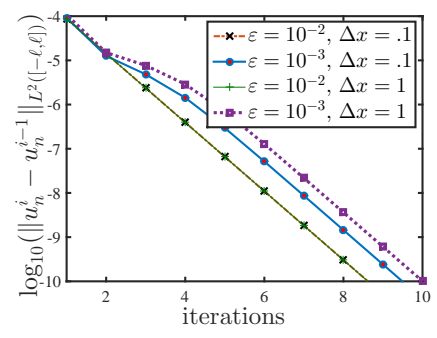

(b)

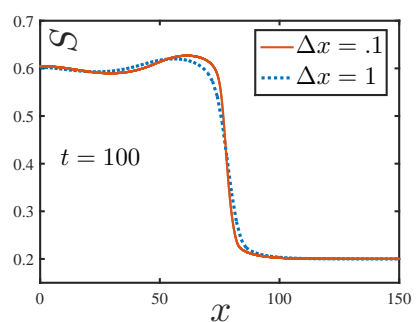

(c)

Figure 17: Convergence study for the hysteresis model. The parameters are $S_{B}=0.2, S_{T}=0.6$, $\Delta x=.1, \Delta t=10^{-3}, \varepsilon=10^{-3}$ unless specified otherwise.

(a) $x_{f}$ as a function of $t$, where $x_{f}$ is the $x$-location at which $S\left(x_{f}, t\right)=\frac{1}{2}\left(S_{B}+S_{T}\right)$. According to (2.19) the TW speed should be $c=\frac{d x_{f}}{d t}=0.8$. From the figure we get $\frac{d x_{f}}{d t}=0.7892$.

(b) Error $\left(\log _{10}\left(\left\|u_{n}^{i}-u_{n}^{i-1}\right\|_{L^{2}([-\ell, \ell])}\right)\right)$ vs iterations for different $\varepsilon$ and $\Delta x$ pairs.

(c) Grid-independence study: $S$ vs $x$ for $\Delta x=1$ and $\Delta x=.1$ at $t=100$.

We conducted a convergence test to ensure that the scheme proposed in Section 5.1 indeed gives the correct results. Some of the findings of the study are shown in Figure 17. Firstly 
we matched the speed of propagation of the profile with the Rankine-Hugoniot speed given in (2.19). The profile speed is calculated from the rate of change of the point $x=x_{f}$ at which $S\left(x_{f}, t\right)=\frac{1}{2}\left(S_{B}+S_{T}\right)$. By $(2.20), \zeta=0$ at this point, meaning that $x_{f}=c t$. The result for the hysteresis case is shown in Figure 17a, and the speeds matched very well for all the cases. This proves that the traveling wave assumption in (2.13) is correct.

Next a convergence study was done for different $\varepsilon$ and $\Delta x$ pairs where the $L^{2}([-\ell, \ell])$ errors of consecutive pressure iterates were plotted against inner iterations. Figure $17 \mathrm{~b}$ shows that the errors are decreasing in all the cases in a linearly convergent fashion. Finally Figure 17c shows the grid independence of the results. With this we start discussing the capillary hysteresis case.

\section{Capillary Hysteresis}
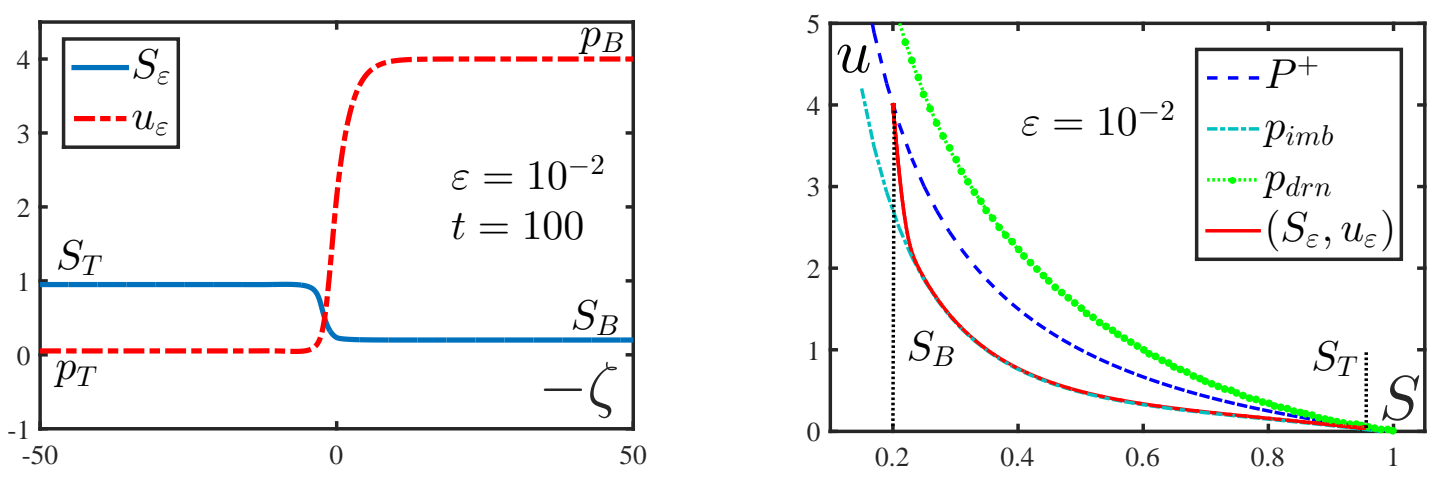

Figure 18: $S_{\varepsilon}$ and $u_{\varepsilon}$ vs. $-\zeta$ (left); and $u_{\varepsilon}$ vs. $S_{\varepsilon}$ (right) for the hysteresis model in the limit $S_{T} \uparrow 1$. The figures are obtained for $S_{T}=.97, S_{B}=0.2, \Delta x=.1, \Delta t=10^{-3}$.

The case $S_{T}=1$ was studied first. To avoid degeneracy $S_{T}$ is kept slightly less than 1 . The monotone profiles of $S$ and $u$, shown in Figure 18, agrees with the theory for $S_{T}=1$.

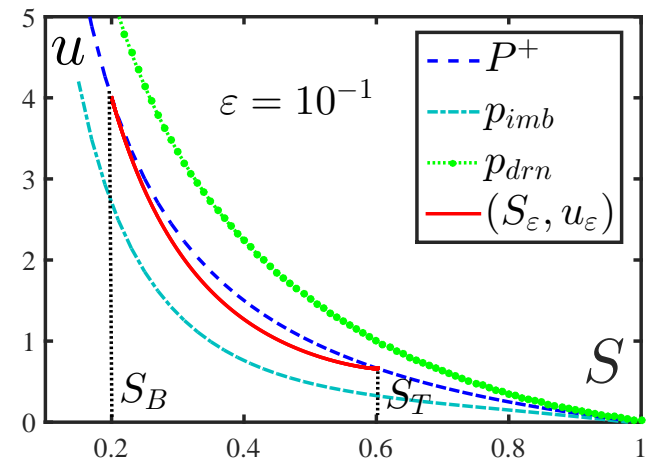

Figure 19: $u_{\varepsilon}$ vs. $S_{\varepsilon}$ for $S_{B}=0.2, S_{T}=0.6$, $\Delta x=.1, \Delta t=10^{-3}$ and $\varepsilon=10^{-1}$.
Next we move on to the $S_{T}<1$ case. We fix $S_{T}=0.6$ and $S_{B}=0.2$ and vary $\varepsilon$. Figure 19 shows the result for $\varepsilon=10^{-1}$. The orbit is monotone and $E_{T}$ is a stable sink. However from Theorem $3.2 \mathrm{~b}$ we expect $E_{T}$ to become a stable spiral sink as $\varepsilon$ becomes small enough. This is indeed the case, as seen from Figure 20. For $\varepsilon=10^{-2}$ we clearly see that there is a stable spiral around $E_{T}$. This implies that the $\varepsilon_{m}$ for Theorem 3.2b is in between .1 and .01. Consequently $u_{\varepsilon}$ and $S_{\varepsilon}$ profiles, as a function of $-\zeta$, are non-monotone. 

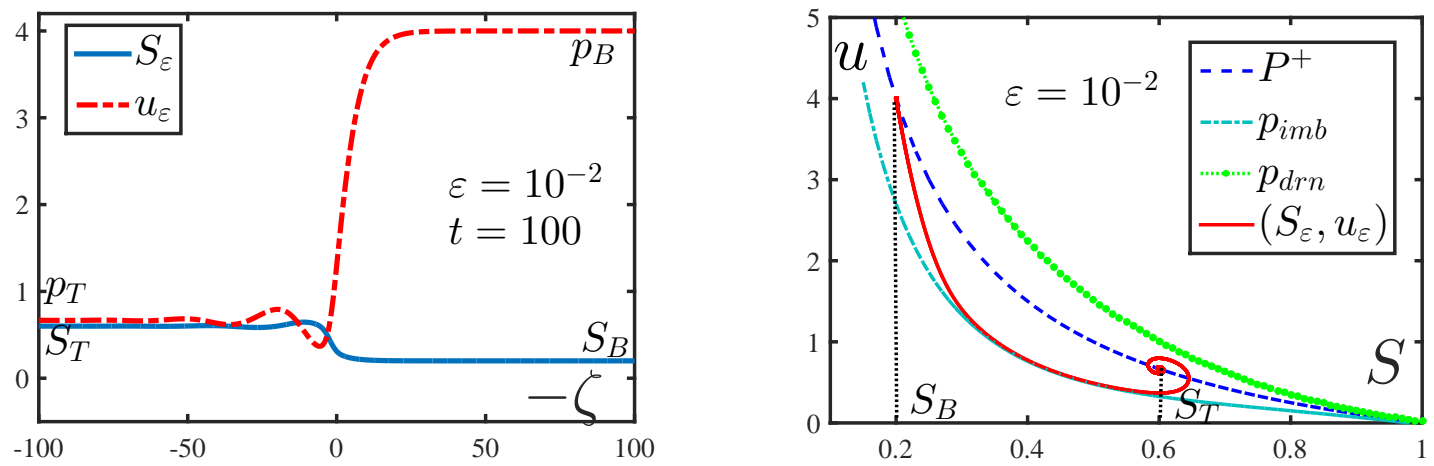

Figure 20: $S_{\varepsilon}$ and $u_{\varepsilon}$ vs. $-\zeta$ (left); and $u_{\varepsilon}$ vs. $S_{\varepsilon}$ (right) for $\varepsilon=10^{-2}$. The other parameters are $S_{B}=0.2, S_{T}=0.6, \Delta x=.1, \Delta t=10^{-3}$.

Similar figures but for $\varepsilon=10^{-3}$ are shown in Figure 21. It is important to mention that longer times are required for the travelling wave profile to develop for the $\varepsilon=10^{-3}$ case in comparison to the $\varepsilon=10^{-2}$ case. This is because if $p_{i m b}(S)<u<p_{d r n}(S)$ then $\partial_{t} S=$ $H_{\varepsilon}\left(\frac{P^{+}(S)-u}{P^{-}(S)}\right) \approx \varepsilon H_{1}\left(\frac{P^{+}(S)-u}{P^{-}(S)}\right)$. So the time required for a profile to develop to a travelling wave profile, scales with $\frac{1}{\varepsilon}$. Also note that the part close to $S=S_{B}$ between the imbibition and drainage curves is much steeper for $\varepsilon=10^{-3}$. This supports Corollary 3.1.
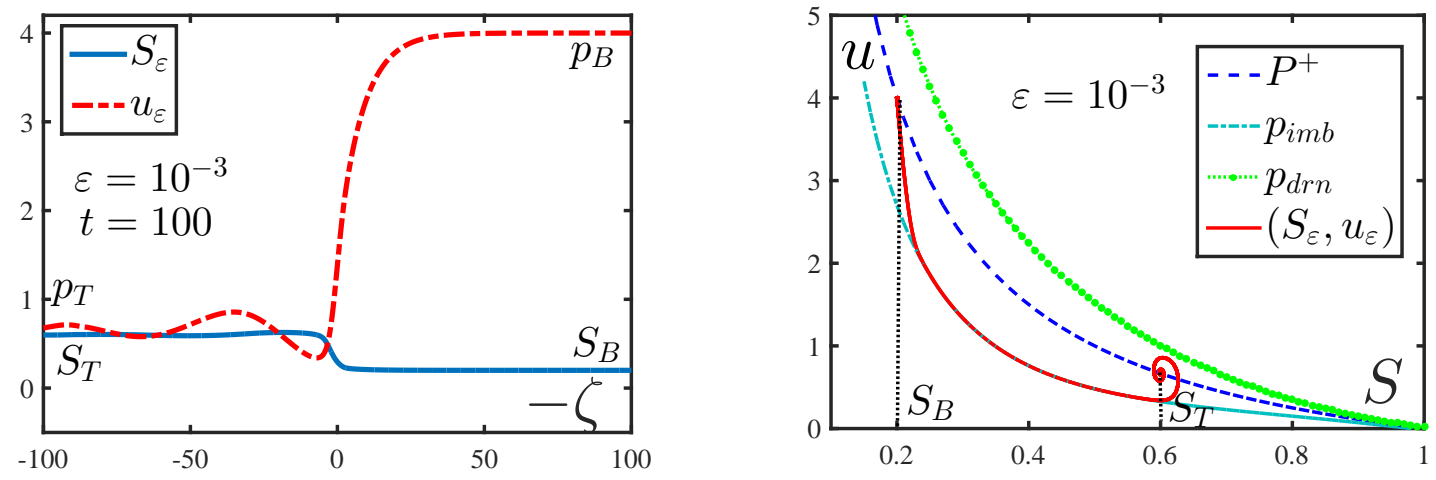

Figure 21: Results for $\varepsilon=10^{-3}$. The other parameters are as in Figure 20.

Another observation is that the period of oscillations in the $S$ and $u$ profiles are wider for $\varepsilon=10^{-3}$. This is a direct consequence of Remark 3.1. The period of oscillation scales with $\mathcal{O}\left(\varepsilon^{-1 / 2}\right)$. Lastly it is seen that the overshoot for $\varepsilon=10^{-3}$ is less than $\varepsilon=10^{-2}$. This follows from Proposition 3.5. In the $S$ - $u$ phase plane the $S$-range of the spiral shrinks with decrease in $\varepsilon$. So the overshoot gets shallower. Consequently, the numerical results reproduce all our predictions for hysteresis. 


\section{Dynamic Capillarity}
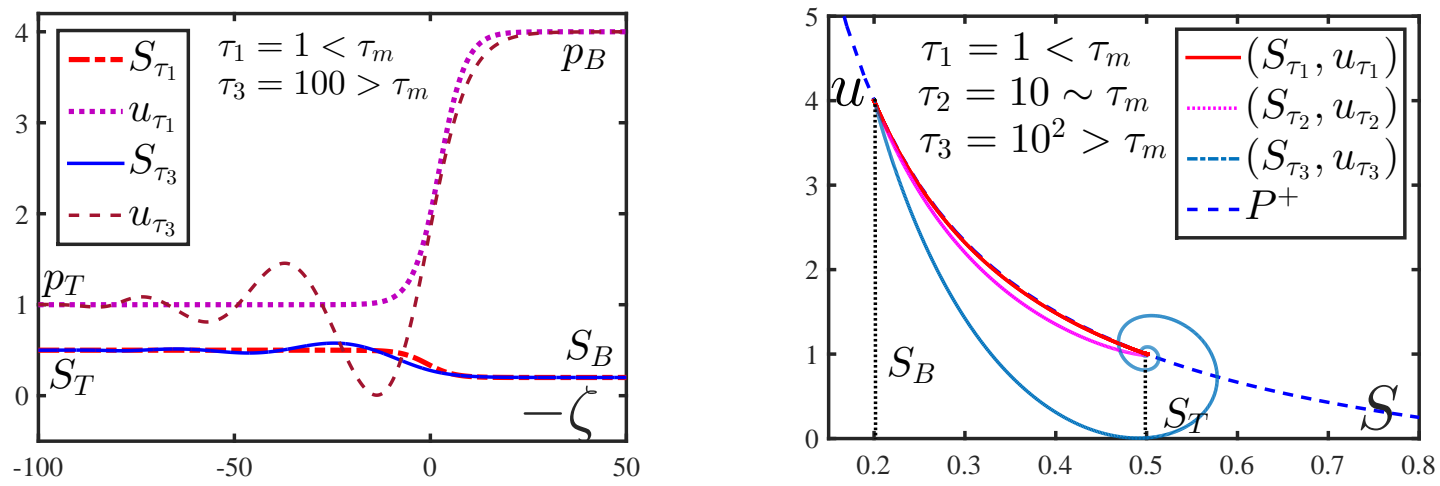

Figure 22: $S_{\tau}$ and $u_{\tau}$ vs. $-\zeta$ (left); and $u_{\tau}$ vs. $S_{\tau}$ (right) for different $\tau$ values. The $\tau$ values used are $\tau_{1}=1, \tau_{2}=10$ and $\tau_{3}=100$. The other parameters are $S_{B}=0.2, S_{T}=0.5, \Delta x=.1$, $\Delta t=10^{-3}$. In this case $\alpha\left(S_{B}, S_{T}\right)>0$.

The $P^{+}(S)$ and $k(S)$ functions are as in (5.6). We first take $f(S)=1$. Figure 22 depicts the case $\alpha\left(S_{B}, S_{T}\right)>0$ with $S_{B}=0.2$ and $S_{T}=0.5$. As expected from the theory, for $\tau<\tau_{m} S$ and $u$ profiles are monotone but for $\tau>\tau_{m}$ they become non-monotone as $E_{T}$ becomes a spiral sink. $\tau_{3}$ is taken so that $w_{\tau_{3}}\left(S_{T}\right) \approx 0$ and hence $\tau_{3} \approx \tau_{*}$. Also a case with $\tau \sim \tau_{m}$ is shown which has no overshoot and the orbit goes directly to $E_{T}$. These behaviours agree with the results presented in Propositions 4.2,4.3 and 4.4.
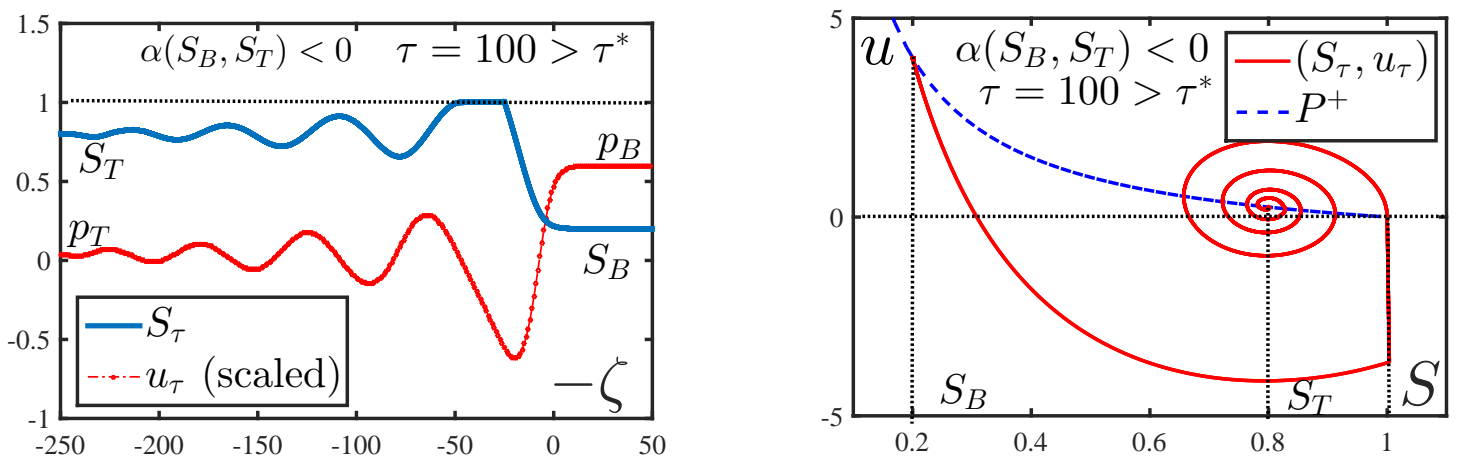

Figure 23: $S_{\tau}$ and $u_{\tau}$ vs. $-\zeta$ (left); and $u_{\tau}$ vs. $S_{\tau}$ (right) for $\alpha\left(S_{B}, S_{T}\right)<0$ and $\tau=100>\tau^{*}$. Here $S_{B}=0.2, S_{T}=0.8 . \delta=.001$ has been used in $P_{e}^{\delta}(4.19)$.

Next we investigate the case when $\alpha\left(S_{B}, S_{T}\right)<0$ with $S_{B}=0.2$ and $S_{T}=0.8$. Figure 23 shows the profiles and orbits for $\tau>\tau^{*}$. A value $\delta=.001$ has been used for $P_{e}^{\delta}$ (see (4.19)). The orbit behaves exactly as predicted. The pressure remains continuously differentiable with $\zeta$ but saturation has a plateau at $S=1$. This means that, in the limit, the extension eliminates the possibility of $S>1$ which in turn eliminates unphysical solutions.

Finally, we investigate the effect of $f \notin L^{1}\left(\left[S_{B}, 1\right]\right)$. We take the most general case given by Remark 4.3, and choose $f(S)$ of the form $f(S)=\frac{1}{S_{\beta}-S}$ with $S_{B}=0.2, S_{T}=0.6$ and $S_{\beta}=0.8$. Figure 24 displays the results. The situation remains mostly unchanged except close to $S_{\alpha}$ the orbits move in a restricted way. The saturation indeed stays lower than $S_{\alpha}$ but for high 
enough $\tau$ values it approaches $S_{\alpha}$. This is in good agreement with our theoretical predictions in Theorem 4.4 and Corollary 4.1.
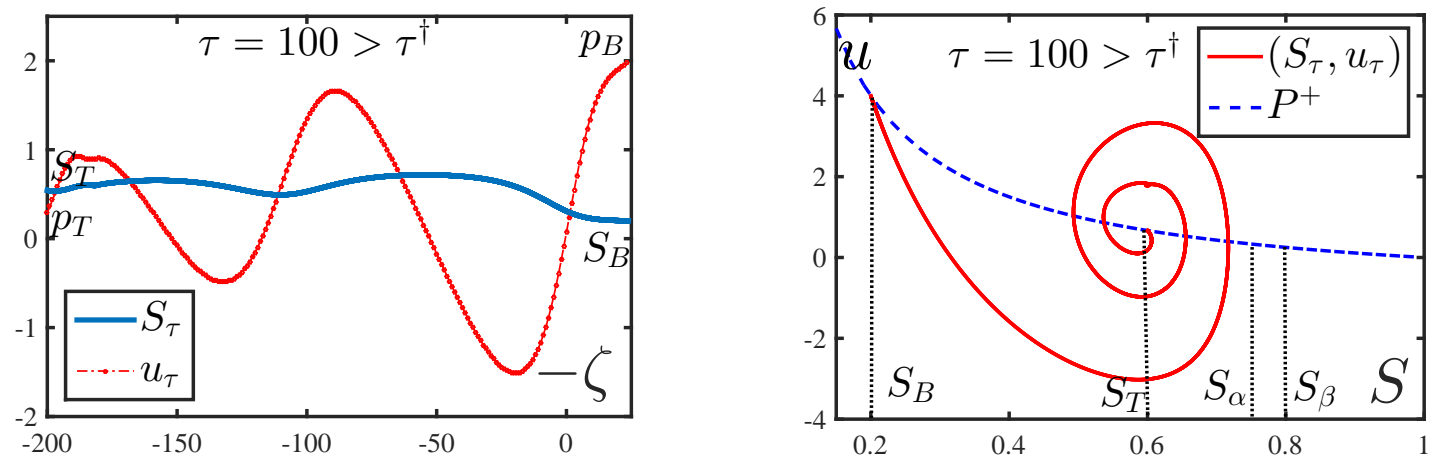

Figure 24: $S_{\tau}$ and $u_{\tau}$ vs. $-\zeta$ (left); and $u_{\tau}$ vs. $S_{\tau}$ (right) for the case $f \notin L^{1}\left(\left[S_{B}, 1\right]\right)$. Here $f(S)=\frac{1}{S_{\beta}-S}$ with $S_{B}=0.2, S_{T}=0.6$ and $S_{\beta}=0.8$. Here $\tau=100>\tau_{*}$.

\section{Conclusion}

In this paper we discussed the implications of including non-equilibrium effects in unsaturated porous flow models. Specifically, the play-type hysteresis and dynamic capillarity effects are considered in the saturation-pressure relationship. One focus was on analysing the occurrence of non-monotone saturation or pressure profiles (overshoots) arising due to non-equilibrium effects. To this end, the traveling wave analysis is considered to understand the flow in a long, homogeneous vertical porous column.

The analysis is done first for hysteresis models. In this case, the existence of travelling wave solutions was shown first for the regularized case and then for the limiting case, leading to a playtype hysteresis model. It was proved that overshoots may appear in the regularised hysteresis models, which correspond to non-vertical scanning curves. However, in the limit situation these overshoots can disappear and the saturation-pressure orbits lie on the imbibition curve.

Next we have investigated the dynamic capillarity effects, for which the existence of TW solutions is proved. Furthermore, the existence of a threshold value for the dynamic capillarity parameter is shown so that for values less than this the travelling waves are monotone, but become non-monotone for values above the threshold. Moreover, similar thresholds are found for the dynamic capillary parameter that dictates whether the overshoot will have regions of positive pressure or whether the overshoot will reach a maximum corresponding to the full saturation. Also mechanisms to restrict saturation to physically relevant values and to include residual saturation were analysed.

Finally, an implicit numerical scheme to solve the non-linear, pseudo parabolic equations corresponding to the non-equilibrium model was proposed. For solving the emerging time discrete, non-linear equations, an L-scheme was used, and the scheme converges irrespective of initial guess. This scheme is used for solving the original partial differential equation in a large, but finite domain. The numerical results converge to the travelling wave profiles, as predicted by the theoretical analysis. 


\section{Acknowledgment}

C.J. van Duijn acknowledges the support of the Darcy Centre of Utrecht University and Eindhoven University of Technology. K. Mitra is supported by Shell and the Netherlands Organisation for Scientific Research (NWO) through the CSER programme (project 14CSER016) and by the Hasselt University through the project BOF17BL04. The research of I.S. Pop is supportde by the Research Foundation-Flanders (FWO) through the Odysseus programme (project G0G1316N).

\section{References}

[1] Abreu, E., Vieira, J., Computing numerical solutions of the pseudo-parabolic BuckleyLeverett equation with dynamic capillary pressure, Mathematics and Computers in Simulation 137 (2017): 29-48

[2] Bedjaoui, N., LeFloch, P.G., Diffusive-dispersive traveling waves and kinetic relations. I. Nonconvex hyperbolic conservation laws. J. Differential Equations 178 (2002) 574-607.

[3] Beliaev, A. Y and Hassanizadeh, S. M., A theoretical model of hysteresis and dynamic effects in the capillary relation for two-phase flow in porous media, Transp. in Porous Media 43 (2001): 487 - 510

[4] Beliaev, A. Y. and Schotting, R. J. , Analysis of a new model for unsaturated flow in porous media including hysteresis and dynamic effects, Comput. Geosci. 5 (2001): 345-368

[5] Bertsch, M., Smarrazzo, F. and Tesei, A., Pseudoparabolic regularization of forwardbackward parabolic equations: a logarithmic nonlinearity. Anal. PDE 6 (2013): 17191754 .

[6] Böhm, M. and Showalter, R.E., A nonlinear pseudoparabolic diffusion equation. SIAM J. Math. Anal. 16 (1985): 980-999.

[7] Bottero, S., Hassanizadeh, S.M., Kleingeld, P.J. and Heimovaara, T.J., Nonequilibrium capillarity effects in two-phase flow through porous media at different scales, Water Resour. Res. 47 (2011), W10505

[8] Bourgeat, A. and Panfilov, M., Effective two-phase flow through highly heterogeneous porous media: Capillary nonequilibrium effects, Comput. Geosci. 2 (1998): 191-215.

[9] Brenner, K., Cancès, C., Hilhorst, D., Finite volume approximation for an immiscible two-phase flow in porous media with discontinuous capillary pressure, Comput. Geosci. 17 (2013): 573-597.

[10] Brokate, M., Botkin, N.D., Pykhteev, O.A., Numerical simulation for a two-phase porous medium flow problem with rate independent hysteresis, Physica B 407 (2012): 1336-1339.

[11] Cancès, C., Choquet, C. , Fan, Y. and Pop, I.S., An existence result related to two-phase flows with dynamical capillary pressure, CASA Report 10-75, Eindhoven University of Technology, 2010.

[12] Cao, X. and Pop, I.S., Degenerate two-phase porous media flow model with dynamic capillarity, J. Differential Equations 260 (2016): 2418-2456. 
[13] Cao, X. and Pop, I.S., Two-phase porous media flows with dynamic capillary effects and hysteresis : uniqueness of weak solutions. Comput. Math. Appl., 69(2015): 688-695.

[14] Cao, X. and Pop, I.S., Uniqueness of weak solutions for a pseudo-parabolic equation modeling two phase flow in porous media, Appl. Math. Letters 46 (2015): 25-30

[15] Collins, R.E., Flow of fluids through porous materials, Petroleum Publishing Co., (1976)

[16] Corli, A., Rohde, C. and Schleper, V., Parabolic approximations of diffusive-dispersive equations. J. Math. Anal. Appl. 414 (2014): 773-798.

[17] Cuesta, C., van Duijn, C. J. and Hulshof, J., Infiltration in porous media with dynamic capillary pressure: travelling waves, Eur. J. Appl. Math. 11 (2000): 381-397

[18] DiCarlo, D. A. , Experimental measurements of saturation overshoot on infiltration. Water Resour. Res. 40 (2004): W04215, doi:10.1029/ 2003WR002670.

[19] DiCarlo, D. A., Juanes, R., LaForce, T., Witelski, T.P., Nonmonotonic traveling wave solutions of infiltration into porous media, Water Resour. Res. 44 (2008): W02406, doi:10.1029/2007WR005975

[20] Doster, F., Zegeling, P., Hilfer, R., Numerical solutions of a generalized theory for macroscopic capillarity, Phys. Rev. E 81 (2010), 036307

[21] Egorov, A. G., Dautov, R. Z., Nieber, J. L. and Sheshukov, A. Y., Stability analysis of gravity-driven infiltrating flow, Water Resour. Res. 39 (2003): 1266, doi:10.1029/2002WR001886.

[22] Hassanizadeh, S. M. and Gray, W. G., Thermodynamic basis of capillary pressure in porous media, Water Resour. Res. 29 (1993): 3389-3405

[23] Helmig, R., Weiss, A. and Wohlmuth, B. I., Dynamic capillary effects in heterogeneous porous media, Comput. Geosci. 11 (2007): 261-274

[24] Hilfer, R. and Steinle, R., Saturation overshoot and hysteresis for two phase flow in porous media, Eur. Phys. J. Spec. Top. 223(2014): 2323

[25] Koch, J. , Rätz, A. and Schweizer, B., Two-phase flow equations with a dynamic capillary pressure, Eur. J. Appl. Math. 24 (2013): 49-75.

[26] Manthey, S., Hassanizadeh, S. M., Helmig, R., Hilfer , R., Dimensional analysis of twophase flow including a rate-dependent capillary pressure-saturation relationship, Adv. Water Res. 31 (2008): 1137-1150

[27] Milisic, J.P., The unsaturated flow in porous media with dynamic capillary pressure, arXiv:1703.10662

[28] Mikelić, A., A global existence result for the equations describing unsaturated flow in porous media with dynamic capillary pressure, J. Differential Equations 248 (2010): 1561-1577.

[29] Morrow, N. R. and Harris, C. C., Capillary equilibrium in porous materials, Soc. Pet. Eng. Jour. (1965)

[30] Niessner, J. and Hassanizadeh, S. M. , A model for two-phase flow in porous media including fluid-fluid interfacial area, Water Resour. Res. 44 (2008) 
[31] Nordbotten, J. M. and Celia, M. A. ,Geological Storage of CO2: Modeling Approaches for Large-Scale Simulation, Wiley (2011)

[32] Papafotiou, A., Sheta, H. and Helmig, R., Numerical modeling of two-phase hysteresis combined with an interface condition for heterogeneous porous media. Comput. Geosci. 14 (2010): 273-287.

[33] Parker, J.C. and Lenhard, R.J., A Model for hysteretic constitutive relations governing multiphase flow 1. Saturation pressure relations. Water Resour. Res. 23 (1987): 618-624

[34] Plohr,B., Marchesin, D., Bedrikovetsky, P. and Krause, P., Modeling hysteresis in porous media flow via relaxation, Comput. Geosci. 5 (2001): 225-256

[35] Pop, I. S., van Duijn, C.J., Niessner, J. and Hassanizadeh, S. M. , Horizontal redistribution of fluids in a porous medium: The role of interfacial area in modeling hysteresis, Adv. Water Res. 32 (2009), 383-390.

[36] Pop, I. S., Radu, F. and Knabner, P., Mixed finite elements for the Richards' equation: linearization procedure, J. Comput. Appl. Math. 168 (2004), 365-373.

[37] Quarteroni, A. Sacco, R. and Saleri, F., Numerical Mathematics, Springer-Verlag, 2000.

[38] Radu, F., Kumar, K., Nordbotten, J.M. and Pop, I. S., A robust, mass conservative scheme for two-phase flow in porous media including Hölder continuous nonlinearities, IMA J. Numer. Anal. (2017) drx032. doi: 10.1093/imanum/drx032.

[39] Rätz, A. and Schweizer, B., Hysteresis models and gravity fingering in porous media, Z. Angew. Math. Mech. 94 (2014), 645-654.

[40] Schweizer, B., Homogenization of degenerate two-phase flow equations with oil-trapping. SIAM J. Math. Anal. 39 (2008), 1740-1763.

[41] Schweizer, B., The Richards equation with hysteresis and degenerate capillary pressure, J. Differential Equations 252 (2012), 5594-5612.

[42] Schweizer, B., Instability of gravity wetting fronts for Richards equations with hysteresis, Interfaces Free Bound. 14 (2012), no. 1, 37-64

[43] Schweizer, B., Laws for the capillary pressure in a deterministic model for fronts in porous media, SIAM J. Math. Anal. 36(5) (2005): 1489-1521

[44] Shearer, M., Spayd, K. R. and Swanson E. R., Traveling waves for conservation laws with cubic nonlinearity and BBM type dispersion, J. Differential Equations 259 (2015) $3216-3232$

[45] Szymkiewicz, A., Modelling water flow in unsaturated porous media, Springer (2013)

[46] Van Duijn, C. J. and Knabner, P., Travelling waves during the transport of reactive solute in porous media: combination of Langmuir and Freundlich isotherms, Adv. Water Res. 16 (1993): 97-105

[47] Van Duijn, C. J., Fan, Y., Peletier, L.A. and Pop, I. S., Travelling wave solutions for degenerate pseudo-parabolic equations modelling two-phase flow in porous media, Nonlinear Anal. Real World Appl. 14 (2013), 1361-1383. 
[48] Van Duijn, C. J., Peletier, L.A. and Pop, I. S., A new class of entropy solution of the Buckley-Leverett equation, SIAM J. Math. Anal. 39(2) (2007): 507-536

[49] Van Genuchten, M. T., A closed form equation for predicting the hydraulic conductivity of unsaturated soils, Soil. Sci. Soc. Am. J. 44 (1980): 894-897

[50] Visintin, A., Differential model of hysteresis, Springer, (1994)

[51] Zhang, H., Zegeling, P.A., A Numerical Study of Two-Phase Flow Models with Dynamic Capillary Pressure and Hysteresis, Transp. Porous Med 116 (2017):825-846

[52] Zhuang, L., Advanced theories of water redistribution and infiltration in porous media: Experimental studies and modeling. PhD thesis, UU Dept. of Earth Sciences, (2017) 


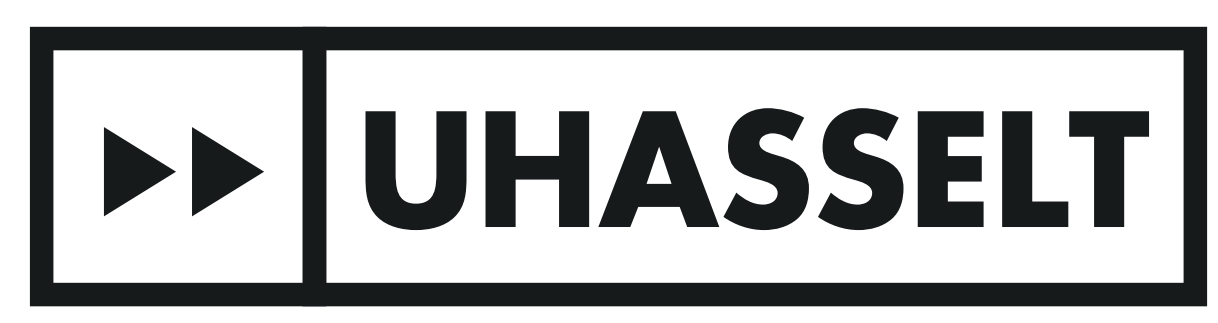

\section{UHasselt Computational Mathematics Preprint Series}

\section{7}

UP-17-05 Cornelis Johannes van Duijn and Koondanibha Mitra, Hysteresis and Horizontal Redistribution in Porous Media, 2017

UP-17-04 Jonas Zeifang, Klaus Kaiser, Andrea Beck, Jochen Schütz and ClausDieter Munz, Efficient high-order discontinuous Galerkin computations of low Mach number flows, 2017

UP-17-03 M.M. Bosschaert, S.G. Janssens, and Yu.A. Kuznetsov, Switching to nonhyperbolic cycles from codim-2 bifurcations of equilibria in DDEs, 2017

UP-17-02 Jochen Schütz, David C. Seal and Alexander Jaust, Implicit multiderivative collocation solvers for linear partial differential equations with discontinuous Galerkin spatial discretizations, 2017

UP-17-01 Alexander Jaust and Jochen Schütz, General linear methods for time-dependent PDEs, 2017

\section{6}

UP-16-06 Klaus Kaiser and Jochen Schütz, A high-order method for weakly compressible flows, 2016

UP-16-05 Stefan Karpinski, Iuliu Sorin Pop, Florin A. Radu, A hierarchical scale separation approach for the hybridized discontinuous Galerkin method, 2016

UP-16-04 Florin A. Radu, Kundan Kumar, Jan Martin Nordbotten, Iuliu Sorin $P o p$, Analysis of a linearization scheme for an interior penalty discontinuous Galerkin method for two phase flow in porous media with dynamic capillarity effects , 2016 
UP-16-03 Sergey Alyaev, Eirik Keilegavlen, Jan Martin Nordbotten, Iuliu Sorin Pop, Fractal structures in freezing brine, 2016

UP-16-02 Klaus Kaiser, Jochen Schütz, Ruth Schöbel and Sebastian Noelle, A new stable splitting for the isentropic Euler equations, 2016

UP-16-01 Jochen Schütz and Vadym Aizinger, A hierarchical scale separation approach for the hybridized discontinuous Galerkin method, 2016

All rights reserved. 